\title{
RETARDING THE DETERIORATION OF WINTER MATURING GUAVA(Psidium guajava L.) FRUITS DURING STORAGE BY POSTHARVEST TREATMENTS OF CALCIUM CHLORIDE AND HYDROGEN PEROXIDE
}

(Received: 6. 3.2018)

\author{
By \\ M. A. A. Mohamed and A. F. Abd El-khalek* \\ Fruit Handling Department, Horticulture Research Institute,Agriculture Research Center, Giza, Egypt. \\ * Horticulture Department, Faculty of Agriculture, Tanta University, Tanta, Egypt.
}

\begin{abstract}
Guavas are perishable fruits and have relatively short marketing life after harvest. The present experiment was conducted in 2015 and 2016 seasons to evaluate the effect of calcium chloride $\left(\mathrm{CaCl}_{2}\right)$, hydrogen peroxide $\left(\mathrm{H}_{2} \mathrm{O}_{2}\right)$ and their combinations as postharvest treatments on quality attributes of 'Etmany' winter maturing guavas during cold storage. All treated fruits were stored at $8 \pm 1^{\circ} \mathrm{C}$ and $90 \pm 5 \%$ relative humidity (RH) for 21 days followed by two days of shelf life at ambient conditions $\left(18-23^{\circ} \mathrm{C}\right.$ and $\left.55-65 \% \mathrm{RH}\right)$ as a simulation for marketing period. The changes of physical and chemical attributes of the fruits were determined at weekly intervals followed by two days shelf life. All applied treatments reduced the deterioration of physical and chemical attributes in comparison to the untreated fruits (control). The combined treatments of 2 or $4 \% \mathrm{CaCl}_{2}$ with $\mathrm{H}_{2} \mathrm{O}_{2}$ at 1 or $2 \%$ were superior in retarding the deterioration of fruit quality during storage as compared to the individual treatments and the control. These applications significantly reduced physiological weight loss, reduced decay incidence, maximized marketable percentage and maintained visual appearance score as well as decreased the loss of firmness, hue angle and lightness values. Also, these applications significantly slowed the increase of fruit contents of total soluble solids and total soluble solids/titratable acidity ratio, as well as significantly reduced the rate of losses in vitamin $\mathrm{C}$ and titratable acidity during storage period. Moreover, pre-storage applications of $4 \% \mathrm{CaCl}_{2}$ plus $\mathrm{H}_{2} \mathrm{O}_{2}$ at 1 or $2 \%$ were the most effective in this respect in comparison to the other treatments. Thus, pre-storage treatments of $4 \%$ $\mathrm{CaCl}_{2}$ plus $\mathrm{H}_{2} \mathrm{O}_{2}$ at 1 or $2 \%$ are effective and promising methods for delaying ripening and senescence respect processes as well as retarding the deterioration of postharvest quality of 'Etmany' winter maturing guavas during cold storage at $8^{\circ} \mathrm{C}$ for up to 21 days followed by two days shelf life at 18 $23^{\circ} \mathrm{C}$.
\end{abstract}

Key words: winter maturing guavas, calcium chloride, hydrogen peroxide, pre-storage, decay, fruit quality.

\section{INTRODUCTION}

Guava (Psidium guajava L.) is one of the most well known edible fruits grown widely throughout tropical and subtropical regions worldwide. Guava fruits are good source of vitamins A, B, C and minerals (Pérez-Gutiérrez et al., 2008). They are very popular to the Egyptian consumers due to its affordable price and high nutritional value. In Egypt, the total guava area reached 36.529 feddans, out of which 33.706 feddans are fruiting. They yield about 343.702 tons (Anonymous, 2016). Guava fruit is highly perishable, and bruising easily after harvest because of the delicate outer skin that offers very little protection against injury. Guava is a climacteric fruit, which ripens rapidly after harvest because of the increase in ethylene production and the high respiration rate (Reyes $\&$ Paul, 1995). The climacteric peak of guava fruits is reached after four to five days from harvest followed by the senescence process, which leads to a loss in fruit commercial value (Bashir \& Abu-Goukh, 2003). All of the above mentioned problems necessitate the development of suitable protocol to extend the storage life of guava fruits and maintain the postharvest quality. Various techniques of low cost and high efficiency can be used in this respect; calcium chloride, and hydrogen peroxide pre-storage applications, and fruit storage at low temperature 
have been used in order to slow down ripening and senescence processes, reducing spoilage and maintaining fruit quality (Ismail et al., 2010; Reena, 2016 and Mohamed et al., 2016).

Calcium is an important constituent of plant tissues and has a vital role in maintaining and modulating various cell functions by increasing membrane stability, cell strength and maintaining the cell to cell contact, thus improving the turgidity of cell wall and retarding tissue softening of fruits and vegetables (Demarty et al., 1984 and Diana et al., 2007). In addition, calcium treatment can delay the onset of the ethylene climacteric period and climacteric peak (Shirzadeh et al., 2011 and Reena, 2016).

Calcium treatment enhances tissue developed resistance to fungal infection by stabilizing or strengthening cell walls by making them more resistant to harmful enzymes produced by fungi (Conway et al., 1994 and Botelho et al., 2000). Calcium chloride treatment extends the storage life by maintaining membrane integrity, reducing respiration rate, controlling disease incidence, delaying membrane lipid catabolism and quality retention of guava fruits (Hiwale \& Singh, 2003 and Kumar et al., 2012). In this concern, postharvest application of calcium chloride represents a safe and effective method for reducing ripening and senescence processes, controlling postharvest decay and softening, delaying color change and maintaining internal quality during storage and shelf life of guava fruits (Shaaban, 2006; Ismail et al., 2010; Mahajan et al., 2011; Reena 2016 and Phani et al., 2016).

Hydrogen peroxide $\left(\mathrm{H}_{2} \mathrm{O}_{2}\right)$ is an environmental friendly compound and is known as an antiseptic because of its cytotoxic effects on many bacterial strains (Juven \& Pierson, 1996). Postharvest treatment of $\mathrm{H}_{2} \mathrm{O}_{2}$ has been proposed for disinfecting fruits and vegetables to reduce microbial populations (Sapers \& Simmons, 1998). $\mathrm{H}_{2} \mathrm{O}_{2}$ at low concentrations acts as a messenger molecule involved in adaptive signaling and triggering tolerance against various abiotic stresses (Dat et al., 2000). It is considered a Generally Regarded As Safe (GRAS) as alternative and have regulatory effects on growth, development and quality of fruit (Ismail et al., 2015). Meanwhile, $\mathrm{H}_{2} \mathrm{O}_{2}$ was successfully used to control pathogens and increase the antioxidant enzyme activities without causing loss of physical and chemical quality during storage in wax apple fruits
(Khandaker et al., 2012), oranges (Mohamed et al., 2016). In this concern, immersing 'Maamoura' guavas in $\mathrm{H}_{2} \mathrm{O}_{2}$ for four minutes before cold storage significantly reduced decay incidence, maintained firmness and improved visual appearance as well as preserved the internal chemical quality during cold storage at $8^{\circ} \mathrm{C}$ for 15 days (Ismail et al., 2010). Moreover, immersing 'Valencia' oranges in $2 \% \mathrm{H}_{2} \mathrm{O}_{2}$ can control postharvest decay incidence, reduce weight loss percentage, increase marketable percentage, maintain firmness and preserve the internal quality during storage at $8^{\circ} \mathrm{C}$ for fifteen weeks (Mohamed et al., 2016).

Winter crops of guavas are free from fruit fly infestation and fetch higher price as compared with the main season crops (Boora et al., 2016). The reduction of losses and extension of postharvest life of guava fruits will help increase the market price. Therefore, the present study was conducted on winter 'Etmany' guavas to evaluate the impact of pre-storage application of calcium chloride and hydrogen peroxide separately, or in combinations, on postharvest decay and quality attributes during cold storage at $8 \pm 1{ }^{\circ} \mathrm{C}$ and $90 \pm 5 \%$ relative humidity $(\mathrm{RH})$, followed by two days shelf life ambient condition $\left(18-23^{\circ} \mathrm{C}\right.$ and $\left.55-65 \% \mathrm{RH}\right)$.

\section{MATERIALS AND METHODS}

\subsection{Plant materials and treatments}

The present study was conducted during the two successive seasons (2015 and 2016) on 'Etmany' winter guava fruits. 'Etmany' guava trees are the vegetative clone of seedy guava that were propagated by root cuttings. The trees are grown in a private orchard in El-Qalubia Governorate, Egypt (latitude, 30 $17^{\prime} \mathrm{N}$; longitude, $31^{\circ} 20^{\prime} \mathrm{E}$ ). Trees were about 10 years of age, planted at a spacing of $5 \times 5$ meters apart in a loamy clay soil under flooding irrigation system and subjected to standard agriculture practices as recommended by the Ministry of Agriculture and Land Reclamation, Egypt. Guava fruits were picked at the maturity stage (yellowish green) on the second week of February according to Silva et al. (1998) from almost similar trees. Fruits were apparently uniform in size and free of visible symptoms of infection. In both experimental seasons, fruits were harvested in plastic boxes (15 kg capacity) and immediately transferred to postharvest laboratory at the Horticulture Research Institute, Agriculture Research Center, Giza, Egypt. Once arrived to the laboratory, the fruits were 
thoroughly washed, sorted and defective fruits, including wounded and other disorders, were excluded, where the rest were considered as sound fruits. The sound fruits at the same maturity stage were washed by $0.01 \%$ sodium hypochlorite water solution for two minutes to clean the surface of the fruits and then air dried at room temperature until visible moisture on the fruits surfaces disappeared completely. A total of 1296 clean sound fruits were selected and randomly divided into nine treatments with three replications and each replicate comprised 12 individual fruits $(9$ treatments $\mathrm{x} 4$ storage periods $\times 3$ replicates $\times 12$ fruits). Fruits were dipped into one of the following treatments: (1) distilled water for untreated fruits (control), (2) calcium chloride $\left(\mathrm{CaCl}_{2}\right)$ at $2 \%$, (3) $\mathrm{CaCl}_{2}$ at $4 \%$, (4) hydrogen peroxide $\left(\mathrm{H}_{2} \mathrm{O}_{2}\right)$ at $1 \%$, (5) $\mathrm{H}_{2} \mathrm{O}_{2}$ at $2 \%$, (6) $\mathrm{CaCl}_{2}$ at $2 \%+\mathrm{H}_{2} \mathrm{O}_{2}$ at $1 \%$, (7) $\mathrm{CaCl}_{2}$ at $2 \%+\mathrm{H}_{2} \mathrm{O}_{2}$ at $2 \%,(8) \mathrm{CaCl}_{2}$ at $4 \%+\mathrm{H}_{2} \mathrm{O}_{2}$ at $1 \%$ and (9) $\mathrm{CaCl}_{2}$ at $4 \%+\mathrm{H}_{2} \mathrm{O}_{2}$ at $2 \%$. Posthervest treatments were performed by dipping guava fruits for five minutes in five liter of aqueous solutions containing Tween-80 $0.05 \%(\mathrm{v} / \mathrm{v})$ to improve wettability and adherence to guavas surface, after that fruits were allowed to dry for $30 \mathrm{~min}$ at room temperature by electric fan. After that, the fruits of each treatment were placed into unsealed plastic bags with a thickness of $0.04 \mathrm{~mm}$. and then the bags were packaged in cardboard boxes with dimensions of $45 \times 35 \times 10 \mathrm{~cm}$. All experimental boxes were stored at $8 \pm 1^{\circ} \mathrm{C}$ and $90 \pm 5 \% \mathrm{RH}$ for 21 days. All treatments were examined at harvest time and every seven days intervals of cold storage at $8 \pm 1^{\circ} \mathrm{C}$ plus two days shelf life at ambient conditions $\left(18-23^{\circ} \mathrm{C}\right.$ and $\left.55-65 \% \mathrm{RH}\right)$ as a shelf life period to examine the effect of pre-storage applications on the physical and the chemical characteristics of guava fruits.

\subsection{Measurement of fruit weight loss, decay, marketable percentage and visual appearance}

Weight loss percentage was calculated by the following formula: [(A-B)/A $\times 100]$, where $\mathrm{A}$ is the initial fruit weight and $B$ is the fruit weight after each period of cold storage.

Decay percentage was scored by the number of decayed fruits due to fungus or any microorganisms infection and calculated as a percentage of the initial number of stored fruits using the following formula: [(C/A $) \times 100]$, where $\mathrm{A}$ is the number of stored fruits and $\mathrm{C}$ is the number of decayed fruits after each period of cold storage.
Marketable percentage was calculated by the following equation: [(D/A) $\times 100]$, where $\mathrm{A}$ is the fruit weight before storage and $\mathrm{D}$ is the sound fruit weight after specified storage period.

Visual appearance was measured by a rating system and fruit was scored as very good $=9$, good $=7$, acceptable $=5$, unacceptable $=3$ and poor $=1$

\subsection{Measurement of fruit color and firmness}

Skin color was measured by using a Minolta CR-400 Chroma Meter (Minolta Co .ltd. Osaka, Japan). The color measurement was expressed in chromaticity values of lightness (L) and hue angel $\left(\mathrm{h}^{\circ}\right)$. Three readings were taken at different locations of each guava fruit during each data observation date according to McGuire (1992).

Fruit firmness was measured in three guava fruits per replication at two equatorial sites by using penetrometer (FT-327, Effegi hand-held, facchini, Alfonsine, Italy) equipped with an 8 $\mathrm{mm}$ cylindrical stainless steel plunger tip (Watkins \& Harman, 1981). Two readings were taken on the flesh of each fruit after peeling. The firmness value was expressed in terms of Newton $/ \mathrm{cm}^{2}$.

\subsection{Measurement of contents in vitamin $C$, total soluble solids (TSS), titratable acidity (TA) and TSS/TA ratio in pulp}

The juice was extracted by crushing the pulp of guavas and straining through a muslin cloth. The extracted juice was used for measuring internal fruit quality as follow:

Vitamin C content was estimated according to A.O.A.C. (2000). Samples of fruit juice were used, oxalic acid solution was added to each sample and titrated with 2,6-dichlorophenolindophenol dye solution, and expressed as a milligram of ascorbic acid, and was calculated as $\mathrm{mg} / 100 \mathrm{ml}$ of the juice.

TSS content was measured using a hand refractometer, 0-32 scale (ATAGO N-1 $\mathrm{E}_{\mathrm{E}}$, Japan) and expressed in ${ }^{\circ}$ Brix after making the temperature correction at $20^{\circ} \mathrm{C}$ according to A.O.A.C. (2000).

TA was assayed based on the method of adopting the procedure described by A.O.A.C. (2000). Aliquots of fruit juice were titrated against $0.1 \mathrm{~N} \mathrm{NaOH}$ in the presence of phenolphthalein as an indicator to the end point, and was calculated as grams of citric acid per $100 \mathrm{ml}$ of juice.

TSS/TA ratio was calculated from the values recorded for fruit juice TSS and TA percentages determined. 


\subsection{Experimental design and statistical data analysis}

This experiment was arranged in a completely randomized design having three replicates and consisting of two factors; prestorage treatments and storage periods. Data calculated as percentage were transformed to arcsine of square root before statistical analysis and non-transformed means are shown. The effects of treatments and cold storage period on different attributes were analyzed statistically by analysis of variance (ANOVA) using the MSTAT-C statistical package (M-STAT, 1993). Comparisons between means were done by Duncan's multiple range test (DMRT) at probability $\leq 0.05$.

\section{RESULTS AND DISCUSSION}

3.1. Physical quality attributes of 'Etmany' guava fruits during storage as affected by pre-storage applications of calcium chloride $\left(\mathrm{CaCl}_{2}\right)$, hydrogen peroxide $\left(\mathrm{H}_{2} \mathrm{O}_{2}\right)$ and their combinations

\subsubsection{Fruit weight loss, decay incidence, marketable percentage and visual appearance}

Data in Tables (1,2,3 and 4) demonstrate that, weight loss and decay percentage of 'Etmany' guava fruits gradually and significantly increased with prolonging the cold storage period at $8^{\circ} \mathrm{C}$ plus two days shelf life at ambient temperature $\left(18-23^{\circ} \mathrm{C}\right)$ in both seasons. In addition, guava fruits showed gradual and significant reduction in marketable percentage and visual appearance with the advancement of storage period during the two seasons. The results indicated that, the maximum weight loss $(12.48 \& 11.61 \%)$ and decay percentage (16.78 \& $13.37 \%$ ) of 'Etmany' guavas were observed at the end of storage period in the first and second seasons, respectively .Also, this period recorded the minimum marketable fruits percentage $(73.26 \& 76.92 \%)$ and visual appearance score $(5.81 \& 4.81)$ in 2015 and 2016 seasons, respectively.

Also the data in Tables (1, 2, 3 and 4) reveal that, all pre-storage treatments showed significant lower fruit weight loss and decay percentages than untreated fruits (control). On the other hand, treated fruits showed significant higher percentage of marketable fruits and visual appearance score as compared to the control in both seasons. In addition, dipping guava fruits in 2 or $4 \% \mathrm{CaCl}_{2}$ in combination with $\mathrm{H}_{2} \mathrm{O}_{2}$ at 1 or
$2 \%$ for five minutes were more effective in reducing weight loss and decay percentages. Moreover, these treatments increased the percentage of marketable fruits and visual appearance score than individual treatments during cold storage period at $8^{\circ} \mathrm{C}$ plus two days shelf life at ambient temperature $\left(18-23^{\circ} \mathrm{C}\right)$.

The data also indicated that, pre-storage applications of $4 \% \mathrm{CaCl}_{2}$ in combination with $\mathrm{H}_{2} \mathrm{O}_{2}$ at $2 \%$ followed by $4 \% \quad \mathrm{CaCl}_{2}$ in combination with $\mathrm{H}_{2} \mathrm{O}_{2}$ at $2 \%$ had the most effectiveness in reducing guava fruits deterioration of fruit weight, marketable percentage and visual appearance score as compared to the other treatments. Moreover, these treatments also had the least decay incidence during cold storage plus two days shelf life in the two seasons in comparison to the control.

Pre-storage treatment of 'Etmany' guava fruits with $4 \% \mathrm{CaCl}_{2}$ plus $2 \% \mathrm{H}_{2} \mathrm{O}_{2}$ recorded the least significant weight loss $(4.76 \& 3.81 \%)$ and decay incidence $(3.43 \& 2.73 \%)$ and recorded the highest marketable fruits $(92.08 \& 93.65 \%)$ and visual appearance score $(8.58 \& 8.58)$ in 2015 and 2016 seasons, respectively. Whereas, untreated fruits (control) exhibited the highest weight loss (12.67 \& $11.80 \%)$ and decay incidence $(16.74 \& 13.34 \%)$ and also recorded the lowest marketable fruits $(73.43 \& 77.02 \%)$ and visual appearance score $(5.33 \& 4.92)$ in the first and second seasons, respectively.

The interaction effect between pre-storage applications and storage periods showed significant differences at $p \leq 0.05$ for weight loss, decay, marketable percentage and visual appearance of 'Etmany' guava fruits in both seasons.

The reduction in fruit weight loss and decay percentages by pre-storage applications of $\mathrm{CaCl}_{2}$ and $\mathrm{H}_{2} \mathrm{O}_{2}$ especially $4 \% \mathrm{CaCl}_{2}$ plus 1 or $2 \%$ $\mathrm{H}_{2} \mathrm{O}_{2}$ might be due to their positive role in decreasing water evaporation from the surface of the fruits and enhancing resistant ability of fruits against pathogens as mentioned by Mohamed et al. (2016) and Reena (2016). Consequently, these treatments increased marketable fruit percentage and visual appearance score (Ismail et al., 2010 and Mohamed et al., 2016). In addition, the highest weight loss and decay percentages as well as the lowest marketable percentage and visual appearance score of untreated guavas fruits (control) were due to increased breakdown of fruits associated with 
Table (1): Weight loss percentages of 'Etmany' guava fruits as influenced by calcium chloride and hydrogen peroxide applications during storage time at $8 \pm 1^{\circ} \mathrm{C}$ plus two days shelf life at $18-23^{\circ} \mathrm{C}$

\begin{tabular}{|c|c|c|c|c|c|c|c|c|c|}
\hline \multirow{3}{*}{ Pre-storage applications } & \multicolumn{9}{|c|}{ Storage period (days) } \\
\hline & \multirow{2}{*}{\multicolumn{2}{|c|}{$\mathbf{0}$}} & \multirow{2}{*}{\multicolumn{2}{|c|}{7}} & \multicolumn{2}{|c|}{14} & \multirow[t]{2}{*}{21} & \multicolumn{2}{|c|}{ Means } \\
\hline & & & & & \multicolumn{4}{|c|}{ Season 2015} & \\
\hline Distilled water (control) & $4.44 j$ & j-m & 10.63 & def & 15.04 & b & 20.59 a & 12.67 & $\mathbf{A}$ \\
\hline $2 \%$ calcium chloride $\left(\mathrm{CaCl}_{2}\right)$ & 2.801 & $\operatorname{lm}$ & 6.02 & h-k & 9.95 & d-g & 14.17 bc & 8.23 & $\mathbf{B}$ \\
\hline $4 \% \mathrm{CaCl}_{2}$ & $2.54 \quad 1$ & $\operatorname{lm}$ & 6.04 & h-k & 9.63 & d-g & 12.13 cde & 7.59 & $\mathbf{B C}$ \\
\hline $1 \%$ hydrogen peroxide $\left(\mathrm{H}_{2} \mathrm{O}_{2}\right)$ & 2.831 & $\operatorname{lm}$ & 6.20 & h-k & 12.00 & cde & 14.68 bc & 8.93 & B \\
\hline $2 \% \mathrm{H}_{2} \mathrm{O}_{2}$ & $2.67 \quad 1$ & $\operatorname{lm}$ & 6.18 & h-k & 9.34 & d-g & $12.18 \mathrm{~cd}$ & 7.59 & $\mathbf{B C}$ \\
\hline $2 \% \mathrm{CaCl}_{2}+1 \% \mathrm{H}_{2} \mathrm{O}_{2}$ & 2.341 & $\operatorname{lm}$ & 4.25 & $\mathbf{j}-\mathbf{m}$ & 7.99 & $f-i$ & 10.91 def & 6.37 & $\mathbf{C D}$ \\
\hline $2 \% \mathrm{CaCl}_{2}+2 \% \mathrm{H}_{2} \mathrm{O}_{2}$ & 2.281 & Im & 4.21 & $\mathbf{j}-\mathbf{m}$ & 7.10 & g-j & 10.24 def & 5.96 & DE \\
\hline $4 \% \mathrm{CaCl}_{2}+1 \% \mathrm{H}_{2} \mathrm{O}_{2}$ & 2.15 & $\mathbf{m}$ & 4.17 & $\mathrm{j}$-m & 6.26 & h-k & 9.14 efg & 5.43 & $\mathbf{D E}$ \\
\hline $4 \% \mathrm{CaCl}_{2}+2 \% \mathrm{H}_{2} \mathrm{O}_{2}$ & 2.01 I & $\mathbf{m}$ & 3.47 & klm & 5.28 & i-l & 8.28 fgh & 4.76 & $\mathbf{E}$ \\
\hline \multirow[t]{2}{*}{ Means } & 2.67 & D & 5.68 & $\mathbf{C}$ & 9.18 & B & $12.48 \quad \mathrm{~A}$ & & \\
\hline & \multicolumn{9}{|c|}{ Season 2016} \\
\hline Distilled water (control) & $3.48 \mathrm{i}$ & ijk & 9.74 & cd & 14.19 & $\mathbf{b}$ & $19.80 \quad$ a & 11.80 & $\mathbf{A}$ \\
\hline $2 \%$ calcium chloride $\left(\mathrm{CaCl}_{2}\right)$ & 1.831 & kl & 5.08 & ghi & 9.05 & cde & $13.31 \quad$ b & 7.32 & $\mathbf{B C}$ \\
\hline $4 \% \mathrm{CaCl}_{2}$ & 1.56 & $\mathbf{k l}$ & 5.10 & ghi & 8.73 & de & $11.26 \quad \mathrm{c}$ & 6.66 & $\mathbf{C}$ \\
\hline $1 \%$ hydrogen peroxide $\left(\mathrm{H}_{2} \mathrm{O}_{2}\right)$ & 1.86 & $\mathbf{k l}$ & 5.26 & ghi & 11.12 & c & $13.82 \quad b$ & 8.02 & $\mathbf{B}$ \\
\hline $2 \% \mathrm{H}_{2} \mathrm{O}_{2}$ & 1.701 & $\mathbf{k l}$ & 5.24 & ghi & 8.43 & de & $11.30 \quad$ c & 6.67 & $\mathbf{C}$ \\
\hline $2 \% \mathrm{CaCl}_{2}+1 \% \mathrm{H}_{2} \mathrm{O}_{2}$ & 1.37 & $\mathbf{k l}$ & 3.29 & i-l & 7.07 & efg & 10.02 cd & 5.44 & D \\
\hline $2 \% \mathrm{CaCl}_{2}+2 \% \mathrm{H}_{2} \mathrm{O}_{2}$ & 1.301 & kl & 3.25 & i-l & 6.17 & fgh & 9.35 cde & 5.02 & D \\
\hline $4 \% \mathrm{CaCl}_{2}+1 \% \mathrm{H}_{2} \mathrm{O}_{2}$ & 1.17 & kl & 3.22 & i-l & 5.33 & ghi & 8.24 def & 4.49 & DE \\
\hline $4 \% \mathrm{CaCl}_{2}+2 \% \mathrm{H}_{2} \mathrm{O}_{2}$ & 1.03 & & 2.51 & jkkl & 4.33 & hij & 7.36 efg & 3.81 & $\mathbf{E}$ \\
\hline Means & 1.70 & D & 4.74 & $\mathrm{C}$ & 8.27 & B & $11.61 \mathrm{~A}$ & & \\
\hline
\end{tabular}

Means followed by the same letters within pre-storage applications, storage periods and their interactions in each season are not significantly different at level $P \leq 0.05$ according to DMRT.

Table (2): Decay percentages of 'Etmany' guava fruits as influenced by calcium chloride and hydrogen peroxide applications during storage time at $8 \pm 1^{\circ} \mathrm{C}$ plus two days shelf life at $18-23^{\circ} \mathrm{C}$

\begin{tabular}{|c|c|c|c|c|c|c|c|c|c|c|}
\hline \multirow{3}{*}{ Pre-storage applications } & \multicolumn{10}{|c|}{ Storage period (days) } \\
\hline & \multicolumn{2}{|c|}{$\mathbf{0}$} & \multicolumn{2}{|c|}{7} & \multicolumn{2}{|c|}{14} & \multicolumn{2}{|c|}{21} & \multicolumn{2}{|c|}{ Means } \\
\hline & \multicolumn{10}{|c|}{ Season 2015} \\
\hline Distilled water (control) & $\mathbf{0 . 0 0}$ & $\bar{j}$ & 13.61 & ef & 21.43 & b & $\mathbf{3 1 . 9 3}$ & $\mathbf{a}$ & 16.74 & $\mathbf{A}$ \\
\hline $2 \%$ calcium chloride $\left(\mathrm{CaCl}_{2}\right)$ & $\mathbf{0 . 0 0}$ & $\mathbf{j}$ & 6.99 & hi & 12.73 & efg & 19.46 & bc & 9.79 & B \\
\hline $4 \% \mathrm{CaCl}_{2}$ & $\mathbf{0 . 0 0}$ & $\mathbf{j}$ & 4.54 & $\mathbf{i}$ & 11.54 & fg & 15.76 & de & 7.96 & $\mathbf{C}$ \\
\hline $1 \%$ hydrogen peroxide $\left(\mathrm{H}_{2} \mathrm{O}_{2}\right)$ & $\mathbf{0 . 0 0}$ & j & 6.24 & i & 13.20 & ef & 19.92 & $\mathbf{b}$ & 9.84 & B \\
\hline $2 \% \mathrm{H}_{2} \mathrm{O}_{2}$ & $\mathbf{0 . 0 0}$ & $\mathbf{j}$ & 5.46 & $\mathbf{i}$ & 11.91 & fg & $\mathbf{1 6 . 9 3}$ & cd & 8.58 & $\mathbf{B C}$ \\
\hline $2 \% \mathrm{CaCl}_{2}+1 \% \mathrm{H}_{2} \mathrm{O}_{2}$ & $\mathbf{0 . 0 0}$ & $\mathbf{j}$ & $\mathbf{0 . 0 0}$ & $\overline{\mathbf{j}}$ & 6.23 & i & 13.68 & ef & 4.98 & D \\
\hline $2 \% \mathrm{CaCl}_{2}+2 \% \mathrm{H}_{2} \mathrm{O}_{2}$ & 0.00 & j & $\mathbf{0 . 0 0}$ & j & 5.97 & i & 12.82 & efg & 4.70 & DE \\
\hline $4 \% \mathrm{CaCl}_{2}+1 \% \mathrm{H}_{2} \mathrm{O}_{2}$ & $\mathbf{0 . 0 0}$ & $\mathbf{j}$ & $\mathbf{0 . 0 0}$ & $\mathbf{j}$ & 5.72 & $\mathbf{i}$ & 10.90 & fg & 4.15 & DE \\
\hline $4 \% \mathrm{CaCl}_{2}+2 \% \mathrm{H}_{2} \mathrm{O}_{2}$ & $\mathbf{0 . 0 0}$ & $\mathbf{j}$ & $\mathbf{0 . 0 0}$ & $\mathbf{j}$ & 4.09 & i & 9.63 & gh & 3.43 & $\mathbf{E}$ \\
\hline \multirow[t]{2}{*}{ Means } & 0.00 & D & 4.09 & $\mathbf{C}$ & 10.31 & B & 16.78 & $\mathbf{A}$ & & \\
\hline & \multicolumn{10}{|c|}{ Season 2016} \\
\hline Distilled water (control) & $\mathbf{0 . 0 0}$ & & 10.84 & ef & $\mathbf{1 7 . 0 7}$ & $\mathbf{b}$ & 25.43 & & 13.34 & $\mathbf{A}$ \\
\hline $2 \%$ calcium chloride $\left(\mathrm{CaCl}_{2}\right)$ & $\mathbf{0 . 0 0}$ & $\mathbf{i}$ & 5.57 & $\mathbf{h}$ & 10.14 & $\mathbf{f}$ & 15.50 & bc & 7.80 & B \\
\hline $4 \% \mathrm{CaCl}_{2}$ & $\mathbf{0 . 0 0}$ & $\mathbf{i}$ & 3.62 & $\mathbf{h}$ & 9.19 & fg & $\mathbf{1 2 . 5 5}$ & de & 6.34 & C \\
\hline $1 \%$ hydrogen peroxide $\left(\mathrm{H}_{2} \mathrm{O}_{2}\right)$ & $\mathbf{0 . 0 0}$ & $\mathbf{i}$ & 4.97 & $\mathbf{h}$ & 10.51 & ef & $\mathbf{1 5 . 8 7}$ & $\mathbf{b}$ & 7.84 & B \\
\hline $2 \% \mathrm{H}_{2} \mathrm{O}_{2}$ & $\mathbf{0 . 0 0}$ & $\mathbf{i}$ & 4.35 & $\mathbf{h}$ & 9.49 & fg & 13.49 & cd & 6.83 & $\mathbf{B C}$ \\
\hline $2 \% \mathrm{CaCl}_{2}+1 \% \mathrm{H}_{2} \mathrm{O}_{2}$ & $\mathbf{0 . 0 0}$ & $\mathbf{i}$ & $\mathbf{0 . 0 0}$ & i & 4.96 & h & 10.90 & ef & 3.96 & D \\
\hline $2 \% \mathrm{CaCl}_{2}+2 \% \mathrm{H}_{2} \mathrm{O}_{2}$ & $\mathbf{0 . 0 0}$ & $\mathbf{i}$ & $\mathbf{0 . 0 0}$ & $\mathbf{i}$ & 4.76 & $\mathbf{h}$ & 10.21 & $\mathbf{f}$ & 3.74 & DE \\
\hline $4 \% \mathrm{CaCl}_{2}+1 \% \mathrm{H}_{2} \mathrm{O}_{2}$ & $\mathbf{0 . 0 0}$ & $\mathbf{i}$ & $\mathbf{0 . 0 0}$ & $\mathbf{i}$ & 4.56 & $\mathbf{h}$ & 8.68 & fg & 3.31 & DE \\
\hline $4 \% \mathrm{CaCl}_{2}+2 \% \mathrm{H}_{2} \mathrm{O}_{2}$ & $\mathbf{0 . 0 0}$ & $\mathbf{i}$ & 0.00 & i & 3.26 & $\mathbf{h}$ & 7.67 & $\mathbf{g}$ & 2.73 & $\mathbf{E}$ \\
\hline Means & 0.00 & D & 3.26 & $\mathbf{C}$ & 8.22 & $\mathbf{B}$ & 13.37 & $\mathbf{A}$ & & \\
\hline
\end{tabular}

Means followed by the same letters within pre-storage applications, storage periods and their interactions in each season are not significantly different at level $P \leq 0.05$ according to DMRT. 
Table(3):Marketable percentages of 'Etmany' guava fruits as influenced by calcium chloride and hydrogen peroxide applications during storage time at $8 \pm 1^{\circ} \mathrm{C}$ plus two days shelf life at 18 $23^{\circ} \mathrm{C}$

\begin{tabular}{|c|c|c|c|c|c|c|}
\hline \multirow{3}{*}{ Pre-storage applications } & \multicolumn{4}{|c|}{ Storage period (days) } & \multirow{3}{*}{\multicolumn{2}{|c|}{ Means }} \\
\hline & $\mathbf{0}$ & 7 & 14 & 21 & & \\
\hline & \multicolumn{4}{|c|}{ Season 2015} & & \\
\hline Distilled water (control) & 95.56 a & 77.36 ghi & $66.74 \mathrm{k}$ & $54.06 \quad$ l & $\mathbf{7 3 . 4 3}$ & $\mathbf{F}$ \\
\hline $2 \%$ calcium chloride $\left(\mathrm{CaCl}_{2}\right)$ & 97.20 a & 87.43 cd & 78.59 fgh & $69.74 \mathrm{k}$ & 83.24 & $\mathbf{E}$ \\
\hline $4 \% \mathrm{CaCl}_{2}$ & 97.46 a & 89.67 bc & 80.08 efg & $74.90 \mathrm{ij}$ & 85.53 & $\mathbf{D}$ \\
\hline $1 \%$ hydrogen peroxide $\left(\mathrm{H}_{2} \mathrm{O}_{2}\right)$ & 97.17 a & 87.98 bcd & 76.61 hi & $68.39 \quad \mathrm{k}$ & 82.53 & $\mathbf{E}$ \\
\hline $2 \% \mathrm{H}_{2} \mathrm{O}_{2}$ & 97.33 a & 88.70 bcd & 79.78 fgh & $73.18 \mathrm{j}$ & 84.75 & D \\
\hline $2 \% \mathrm{CaCl}_{2}+1 \% \mathrm{H}_{2} \mathrm{O}_{2}$ & 97.66 a & 95.75 a & $86.06 \mathrm{~d}$ & 76.92 ghi & 89.10 & C \\
\hline $2 \% \mathrm{CaCl}_{2}+2 \% \mathrm{H}_{2} \mathrm{O}_{2}$ & 97.72 a & $95.79 \mathrm{a}$ & 87.27 cd & 78.25 fgh & 89.76 & $\mathbf{B C}$ \\
\hline $4 \% \mathrm{CaCl}_{2}+1 \% \mathrm{H}_{2} \mathrm{O}_{2}$ & 97.85 a & $95.83 \mathrm{a}$ & 88.42 bcd & 80.93 ef & 90.76 & $\mathbf{A B}$ \\
\hline $4 \% \mathrm{CaCl}_{2}+2 \% \mathrm{H}_{2} \mathrm{O}_{2}$ & 97.99 a & \begin{tabular}{|l|}
96.53 \\
\end{tabular} & $90.88 \quad b$ & 82.94 e & 92.08 & $\mathbf{A}$ \\
\hline \multirow[t]{2}{*}{ Means } & $97.33 \mathrm{~A}$ & $90.56 \mathrm{~B}$ & $81.60 \mathrm{C}$ & $73.26 \quad \mathrm{D}$ & & \\
\hline & \multicolumn{4}{|c|}{ Season 2016} & & \\
\hline Distilled water (control) & $96.52 \mathrm{a}$ & 80.61 hij & $71.15 \quad 1$ & $59.81 \mathrm{~m}$ & 77.02 & $\mathbf{G}$ \\
\hline $2 \%$ calcium chloride $\left(\mathrm{CaCl}_{2}\right)$ & $98.17 \mathrm{a}$ & 89.65 bcd & 81.73 ghi & $73.74 \quad 1$ & 85.82 & $\mathbf{E F}$ \\
\hline $4 \% \mathrm{CaCl}_{2}$ & $98.44 \mathrm{a}$ & 91.45 bc & 82.99 fgh & 78.31 jk & $\mathbf{8 7 . 8 0}$ & $\mathbf{D}$ \\
\hline $1 \%$ hydrogen peroxide $\left(\mathrm{H}_{2} \mathrm{O}_{2}\right)$ & 98.14 a & 90.05 bed & 79.71 ijk & $72.55 \quad 1$ & 85.11 & $\mathbf{F}$ \\
\hline $2 \% \mathrm{H}_{2} \mathrm{O}_{2}$ & 98.30 a & 90.64 bcd & 82.81 fgh & $76.92 \mathrm{k}$ & 87.17 & DE \\
\hline $2 \% \mathrm{CaCl}_{2}+1 \% \mathrm{H}_{2} \mathrm{O}_{2}$ & 98.63 a & 96.71 a & 88.14 de & 80.19 hij & 90.92 & $\mathbf{C}$ \\
\hline $2 \% \mathrm{CaCl}_{2}+2 \% \mathrm{H}_{2} \mathrm{O}_{2}$ & 98.70 a & 96.75 a & 89.30 cd & 81.40 ghi & 91.54 & BC \\
\hline $4 \% \mathrm{CaCl}_{2}+1 \% \mathrm{H}_{2} \mathrm{O}_{2}$ & $98.83 \mathrm{a}$ & 96.78 a & 90.40 bed & 83.78 fg & 92.45 & $\mathbf{A B}$ \\
\hline $4 \% \mathrm{CaCl}_{2}+2 \% \mathrm{H}_{2} \mathrm{O}_{2}$ & 98.97 a & 97.49 a & $92.58 \quad b$ & 85.57 ef & 93.65 & $\mathbf{A}$ \\
\hline Means & $98.30 \quad \mathrm{~A}$ & 92.24 B & $84.31 \quad \mathrm{C}$ & $\begin{array}{ll}76.92 & \text { D }\end{array}$ & & \\
\hline
\end{tabular}

Means followed by the same letters within pre-storage applications, storage periods and their interactions in each season are not significantly different at level $P \leq 0.05$ according to DMRT.

Table (4): Visual appearance of 'Etmany' guava fruits as influenced by calcium chloride and hydrogen peroxide applications during storage time at $8 \pm 1^{\circ} \mathrm{C}$ plus two days shelf life at $18-23^{\circ} \mathrm{C}$

\begin{tabular}{|c|c|c|c|c|c|c|c|c|c|c|}
\hline \multirow{3}{*}{ Pre-storage applications } & \multicolumn{10}{|c|}{ Storage period (days) } \\
\hline & \multicolumn{2}{|c|}{$\mathbf{0}$} & \multicolumn{2}{|c|}{7} & \multicolumn{2}{|c|}{14} & \multicolumn{2}{|c|}{21} & \multicolumn{2}{|c|}{ Means } \\
\hline & \multicolumn{10}{|c|}{ Season 2015} \\
\hline Distilled water (control) & 9.00 & $\mathbf{a}$ & 6.33 & ef & 4.331 & h & 1.67 & $\mathbf{i}$ & 5.33 & D \\
\hline $2 \%$ calcium chloride $\left(\mathrm{CaCl}_{2}\right)$ & 9.00 & $\mathbf{a}$ & 8.67 & $\mathbf{a}$ & 6.67 & def & 5.00 & gh & 7.33 & $\mathbf{C}$ \\
\hline $4 \% \mathrm{CaCl}_{2}$ & 9.00 & $\mathbf{a}$ & 9.00 & $\mathbf{a}$ & 7.67 & a-d & 6.33 & ef & 8.00 & $\mathbf{A B}$ \\
\hline $1 \%$ hydrogen peroxide $\left(\mathrm{H}_{2} \mathrm{O}_{2}\right)$ & 9.00 & $\mathbf{a}$ & 8.67 & $\mathbf{a}$ & $6.67 \quad$ & def & 5.00 & gh & 7.33 & $\mathbf{C}$ \\
\hline $2 \% \mathrm{H}_{2} \mathrm{O}_{2}$ & 9.00 & $\mathbf{a}$ & 9.00 & $\mathbf{a}$ & 7.33 & b-e & 5.67 & fg & 7.75 & BC \\
\hline $2 \% \mathrm{CaCl}_{2}+1 \% \mathrm{H}_{2} \mathrm{O}_{2}$ & 9.00 & $\mathbf{a}$ & 9.00 & $\mathbf{a}$ & 8.00 & abc & 6.67 & def & 8.17 & $\mathbf{A B}$ \\
\hline $2 \% \mathrm{CaCl}_{2}+2 \% \mathrm{H}_{2} \mathrm{O}_{2}$ & 9.00 & $\mathbf{a}$ & 9.00 & $\mathbf{a}$ & 8.00 & abc & 7.00 & cde & 8.25 & $\mathbf{A B}$ \\
\hline $4 \% \mathrm{CaCl}_{2}+1 \% \mathrm{H}_{2} \mathrm{O}_{2}$ & 9.00 & $\mathbf{a}$ & 9.00 & $\mathbf{a}$ & 8.33 & $\mathbf{a b}$ & 7.33 & b-e & 8.42 & $\mathbf{A}$ \\
\hline $4 \% \mathrm{CaCl}_{2}+2 \% \mathrm{H}_{2} \mathrm{O}_{2}$ & 9.00 & $\mathbf{a}$ & 9.00 & $\mathbf{a}$ & 8.67 & $\mathbf{a}$ & 7.67 & a-d & 8.58 & $\mathbf{A}$ \\
\hline \multirow[t]{2}{*}{ Means } & 9.00 & $\mathbf{A}$ & 8.63 & $\mathbf{A}$ & $7.30 \quad 1$ & $\mathbf{B}$ & 5.81 & $\mathbf{C}$ & & \\
\hline & \multicolumn{10}{|c|}{ Season 2016} \\
\hline Distilled water (control) & 9.00 & $\mathbf{a}$ & 6.00 & fgh & $3.67 \quad \mathrm{i}$ & & 1.00 & $\mathbf{j}$ & 4.92 & $\mathbf{F}$ \\
\hline $2 \%$ calcium chloride $\left(\mathrm{CaCl}_{2}\right)$ & 9.00 & $\mathbf{a}$ & 8.67 & ab & 6.33 & efg & 5.33 & gh & 7.33 & DE \\
\hline $4 \% \mathrm{CaCl}_{2}$ & 9.00 & $\mathbf{a}$ & 9.00 & $\mathbf{a}$ & 7.33 & b-e & 6.00 & fgh & 7.83 & BCD \\
\hline $1 \%$ hydrogen peroxide $\left(\mathrm{H}_{2} \mathrm{O}_{2}\right)$ & 9.00 & $\mathbf{a}$ & 8.33 & abc & 6.33 & efg & 5.00 & $\mathbf{h}$ & 7.17 & $\mathbf{E}$ \\
\hline $2 \% \mathrm{H}_{2} \mathrm{O}_{2}$ & 9.00 & $\mathbf{a}$ & 9.00 & $\mathbf{a}$ & 7.00 & c-f & 5.33 & gh & 7.58 & CDE \\
\hline $2 \% \mathrm{CaCl}_{2}+1 \% \mathrm{H}_{2} \mathrm{O}_{2}$ & 9.00 & $\mathbf{a}$ & 9.00 & $\mathbf{a}$ & 7.67 & a-d & 6.67 & def & 8.08 & $\mathbf{A B C}$ \\
\hline $2 \% \mathrm{CaCl}_{2}+2 \% \mathrm{H}_{2} \mathrm{O}_{2}$ & 9.00 & $\mathbf{a}$ & 9.00 & $\mathbf{a}$ & 8.00 & a-d & 7.33 & b-e & 8.33 & $\mathbf{A B}$ \\
\hline $4 \% \mathrm{CaCl}_{2}+1 \% \mathrm{H}_{2} \mathrm{O}_{2}$ & 9.00 & $\mathbf{a}$ & 9.00 & $\mathbf{a}$ & $8.00:$ & a-d & 7.67 & a-d & 8.42 & $\mathbf{A B}$ \\
\hline $4 \% \mathrm{CaCl}_{2}+2 \% \mathrm{H}_{2} \mathrm{O}_{2}$ & 9.00 & $\mathbf{a}$ & 9.00 & $\mathbf{a}$ & 8.33 & abc & 8.00 & a-d & 8.58 & $\mathbf{A}$ \\
\hline Means & 9.00 & $\mathbf{A}$ & 8.56 & B & 6.96 & C & 5.81 & D & & \\
\hline
\end{tabular}

Means followed by the same letters within pre-storage applications, storage periods and their interactions in each season are not significantly different at level $P \leq 0.05$ according to DMRT.

higher respiratory rate and cellular metabolic activities (Hiwale \& Singh, 2003 and Reena, 2016), thus the control treatment hasted ripening and senescence processes of guavas. Moreover, increasing weight loss and decay incidence of guavas during storage are attributed to the increase in ripening and senescence processes of guava fruits (Hiwale \& Singh, 2003 and Reena, 
2016). Similar findings were obtained by Hiwale \& Singh (2003), Shaaban (2006), Mahajan et al. (2011), Kumar et al. (2012), Phani et al. (2016), Dohre (2016) and Reena (2016). They reported that, weight loss percentage and decay incidence of guavas were reduced by dipping fruits in $\mathrm{CaCl}_{2}$ treatment in comparison to the untreated fruits (control) during cold storage and shelf life. Furthermore, they illustrated that, fruits weight loss and decay incidence gradually and significantly increased with prolonging of storage period.

In addition, these results are in harmony with the findings of (Ismail et al., 2010) who reported that, dipping 'Maamoura' guava fruits in $2 \%$ $\mathrm{CaCl}_{2}$ or $2 \% \mathrm{H}_{2} \mathrm{O}_{2}$ for four minutes reduced weight loss and decay percentages as well as increased visual appearance score during cold storage at $8^{\circ} \mathrm{C}$ for 15 days in comparison to the untreated fruits. Moreover, weight loss percentage and decay incidence of 'Valencia' oranges were reduced by soaking fruits in $2 \%$ $\mathrm{H}_{2} \mathrm{O}_{2}$ for five minutes and also this application increased marketable fruit percentage as compared to untreated fruits during cold storage at $8^{\circ} \mathrm{C}$ for fifteen weeks (Mohamed et al., 2016). In addition, weight loss and decay percentages gradually increased with the advancement of cold storage period as well as marketable fruits and visual appearance decreased too.

\subsubsection{Fruit firmness}

Fruit firmness is often a major quality characteristic judged by the consumer and it is extremely important in overall product acceptance. Fruit firmness changes during 21 days of cold storage at $8^{\circ} \mathrm{C}$ plus two days shelf life at ambient temperature $\left(18-23^{\circ} \mathrm{C}\right)$ are shown in Table (5). The data in the present study indicate that, a gradual and significant decrease in fruit firmness was observed with the progress of cold storage period at $8^{\circ} \mathrm{C}$ followed by two days shelf life at ambient temperature $\left(18-23^{\circ} \mathrm{C}\right)$ in both seasons. The results indicated that, the minimum flesh firmness of 'Etmany' guavas was recorded at the end of storage period and the values reached of $15.92 \& 14.82 \mathrm{~N} / \mathrm{cm}^{2}$ in the first and second seasons, respectively.

The data in Table (5) also showed that, the firmness values of soaking 'Etmany' guava fruits in $\mathrm{CaCl}_{2}$ and $\mathrm{H}_{2} \mathrm{O}_{2}$ were significantly higher as compared to the untreated fruits (control). In addition, immersing guavas in $\mathrm{CaCl}_{2}$ at 2 or $4 \%$ plus $\mathrm{H}_{2} \mathrm{O}_{2}$ at 1 or $2 \%$ was more effective in maintaining fruit firmness than individual treatments and the control in both seasons. The data in the current experiment indicated that, immersing 'Etmany' guavas in $4 \% \mathrm{CaCl}_{2}$ plus $2 \% \mathrm{H}_{2} \mathrm{O}_{2}$ recorded the highest value of flesh firmness $\left(6.07 \& 5.89 \mathrm{~N} / \mathrm{cm}^{2}\right)$ in 2015 and 2016 seasons. On the contrary, the

Table (5): Firmness (N/cm²) of 'Etmany' guava fruits as influenced by calcium chloride and hydrogen peroxide applications during storage time at $8 \pm 1^{\circ} \mathrm{C}$ plus two days shelf life at $18-23^{\circ} \mathrm{C}$

\begin{tabular}{|c|c|c|c|c|c|c|c|c|c|}
\hline \multirow{3}{*}{ Pre-storage applications } & \multicolumn{9}{|c|}{ Storage period (days) } \\
\hline & $\mathbf{0}$ & & 7 & 7 & 14 & & 21 & & ans \\
\hline & \multicolumn{9}{|c|}{ Season 2015} \\
\hline Distilled water (control) & 6.76 & & 4.09 & h-k & $3.00 \mathrm{~lm}$ & 1.83 & $\mathbf{n}$ & 3.92 & $\mathbf{F}$ \\
\hline $2 \%$ calcium chloride $\left(\mathrm{CaCl}_{2}\right)$ & 6.84 & $\mathbf{a}$ & 4.91 & e-h & 3.92 ijk & 2.44 & mn & 4.53 & DE \\
\hline $4 \% \mathrm{CaCl}_{2}$ & 6.87 & $\mathbf{a}$ & 5.24 & def & $4.41 \quad f-j$ & 2.80 & $\operatorname{lm}$ & 4.83 & D \\
\hline $1 \%$ hydrogen peroxide $\left(\mathrm{H}_{2} \mathrm{O}_{2}\right)$ & 6.83 & a & 4.72 & e-i & $3.57 \quad$ jkl & 2.21 & mn & 4.33 & $\mathbf{E}$ \\
\hline $2 \% \mathrm{H}_{2} \mathrm{O}_{2}$ & 6.86 & $\mathbf{a}$ & 5.18 & def & $4.30 \mathrm{~g}-\mathrm{k}$ & 2.76 & $\operatorname{lm}$ & 4.77 & D \\
\hline $2 \% \mathrm{CaCl}_{2}+1 \% \mathrm{H}_{2} \mathrm{O}_{2}$ & 6.88 & $\mathbf{a}$ & 5.92 & bed & 4.83 e-h & 3.49 & kl & 5.28 & $\mathbf{C}$ \\
\hline $2 \% \mathrm{CaCl}_{2}+2 \% \mathrm{H}_{2} \mathrm{O}_{2}$ & 6.90 & & 6.09 & abc & 5.13 d-g & 3.85 & ijk & 5.49 & $\overline{B C}$ \\
\hline $4 \% \mathrm{CaCl}_{2}+1 \% \mathrm{H}_{2} \mathrm{O}_{2}$ & 6.91 & $\mathbf{a}$ & 6.62 & ab & 5.50 cde & 4.18 & h-k & 5.80 & $\mathbf{A B}$ \\
\hline $4 \% \mathrm{CaCl}_{2}+2 \% \mathrm{H}_{2} \mathrm{O}_{2}$ & 6.93 & $\mathbf{a}$ & 6.85 & $\mathbf{a}$ & $5.78 \mathrm{~cd}$ & 4.71 & e-i & 6.07 & $\mathbf{A}$ \\
\hline \multirow[t]{2}{*}{ Means } & 6.86 & $\mathbf{A}$ & 5.51 & B & $4.49 \mathrm{C}$ & 3.14 & D & & \\
\hline & \multicolumn{9}{|c|}{ Season 2016} \\
\hline Distilled water (control) & 6.57 & $\mathbf{a}$ & 3.97 & h-k & $2.92 \operatorname{lmn}$ & 1.78 & $\mathbf{0}$ & 3.81 & $\mathbf{F}$ \\
\hline $2 \%$ calcium chloride $\left(\mathrm{CaCl}_{2}\right)$ & 6.64 & $\mathbf{a}$ & 4.77 & e-h & 3.81 ijk & 2.37 & no & 4.40 & DE \\
\hline $4 \% \mathrm{CaCl}_{2}$ & 6.68 & $\mathbf{a}$ & 5.09 & def & $4.28 \mathrm{f}-\mathrm{j}$ & 2.72 & Imn & 4.69 & D \\
\hline $1 \%$ hydrogen peroxide $\left(\mathrm{H}_{2} \mathrm{O}_{2}\right)$ & 6.63 & $\mathbf{a}$ & 4.58 & $e-i$ & $3.47 \quad \mathrm{kl}$ & 2.15 & no & 4.21 & $\mathbf{E}$ \\
\hline $2 \% \mathrm{H}_{2} \mathrm{O}_{2}$ & 6.67 & $\mathbf{a}$ & 5.03 & def & $4.18 \mathrm{~g}-\mathrm{k}$ & 2.68 & mn & 4.64 & D \\
\hline $2 \% \mathrm{CaCl}_{2}+1 \% \mathrm{H}_{2} \mathrm{O}_{2}$ & 6.68 & $\mathbf{a}$ & 5.76 & bed & 4.69 e-h & 3.39 & klm & 5.13 & $\mathbf{C}$ \\
\hline $2 \% \mathrm{CaCl}_{2}+2 \% \mathrm{H}_{2} \mathrm{O}_{2}$ & 6.70 & $\mathbf{a}$ & 5.92 & abc & $4.98 \mathrm{~d}-\mathrm{g}$ & 3.75 & jk & 5.34 & BC \\
\hline $4 \% \mathrm{CaCl}_{2}+1 \% \mathrm{H}_{2} \mathrm{O}_{2}$ & 6.71 & $\mathbf{a}$ & 6.43 & ab & 5.35 cde & 4.06 & h-k & 5.64 & $\mathbf{A B}$ \\
\hline $4 \% \mathrm{CaCl}_{2}+2 \% \mathrm{H}_{2} \mathrm{O}_{2}$ & 6.73 & $\mathbf{a}$ & 6.65 & $\mathbf{a}$ & $5.61 \mathrm{~cd}$ & 4.57 & e-i & 5.89 & $\mathbf{A}$ \\
\hline Means & 6.67 & $\mathbf{A}$ & 5.36 & B & $4.37 \mathrm{C}$ & 3.05 & D & & \\
\hline
\end{tabular}

Means followed by the same letters within pre-storage applications, storage periods and their interactions in each season are not significantly different at level $P \leq 0.05$ according to DMRT. 
untreated fruits (control) showed the least firmness value $\left(3.92 \& 3.81 \mathrm{~N} / \mathrm{cm}^{2}\right)$ in the first and second seasons, respectively.

Referring to the interaction effect, there was a significant interaction between pre-storage treatments and storage periods at $p \leq 0.05$ for firmness in both seasons of this study.

Indeed, calcium ion forms stabilize cellular membranes by holding the cells altogether and delaying senescence in horticultural crops (Diana et al., 2007). Therefore, the retention of firmness in 'Etmany' guavas treated with $\mathrm{CaCl}_{2}$, $\mathrm{H}_{2} \mathrm{O}_{2}$ and their combinations, especially those treated with $4 \% \mathrm{CaCl}_{2}$ plus 1 or $2 \% \mathrm{H}_{2} \mathrm{O}_{2}$, could be attributed to the role of these compounds in maintaining cellular organization and obstructing cell wall enzyme activities as documented by Mohamed et al. (2016) and Reena (2016). Thus, these treatments increased wall strength and cell cohesion, thereby retarding tissue softening than the untreated fruits (control) that showed minimum flesh firmness.

In addition, the reduction in flesh firmness of 'Etmany' guavas with the progress of storage could be attributed to the increase in the activities of cell wall hydrolysis enzymes and the degradation in cellular structures (Jain et al., 2003).

The observations in the current investigation are very close to those obtained by Hiwale \& Singh (2003), Shaaban (2006), Mahajan et al. (2011), Kumar et al. (2012), Phani et al. (2016) and Reena (2016) They showed higher firmness by treating guava fruits with $\mathrm{CaCl}_{2}$ as compared to untreated fruits during cold storage and shelf life. Moreover, the authors illustrated that, fruit firmness gradually and significantly decreased with prolonging of storage period. In addition, our results fall in line with the earlier reports of Mohamed et al. (2016) who reported that, dipping 'Valencia' oranges in $\mathrm{H}_{2} \mathrm{O}_{2}$ at $2 \%$ for five minutes reduced the deterioration rate in fruit firmness during cold storage at $8^{\circ} \mathrm{C}$ for fifteen weeks in comparison to untreated fruits.

\subsubsection{Fruit skin color and lightness}

Color is an important factor determining the appearance of fruits and consequently its quality. Skin color development represented as hue angle value (yellowish green, more than 90 and greenish yellow or yellow, less than 90) and brightness represented as lightness. The data in Tables (6 and 7) revealed that, skin color of 'Etmany' guava fruits changed directly from yellowish green to yellow with the progress of cold storage at $8^{\circ} \mathrm{C}$ plus two days shelf life at ambient temperature $\left(18-23^{\circ} \mathrm{C}\right)$ in both seasons. Moreover, lightness values gradually and significantly decreased with prolonging of cold storage period plus two days shelf life during the two seasons in this study. The results showed that, the minimum hue angle and lightness of guavas were recorded at the end of storage period and the values reached of $82.52 \& 78.19$ and $70.36 \& 72.17$, respectively in the first and second seasons, respectively.

Data in Tables (6 and 7) also demonstrated that, dipping 'Etmany' guava fruits in $\mathrm{CaCl}_{2}$, $\mathrm{H}_{2} \mathrm{O}_{2}$ and their combinations significantly delayed skin color development during cold storage period at $8^{\circ} \mathrm{C}$ followed by two days shelf life at ambient temperature $\left(18-23^{\circ} \mathrm{C}\right)$ as compared to untreated guava fruits (control). In addition, these treatments significantly reduced the decrease in brightness than the untreated fruits (control) in both seasons. Thus, all prestorage applications especially combined applications of 'Etmany' guava fruits were still greener and more bright than the untreated fruits (control). Treated 'Etmany' guava fruits with $4 \%$ $\mathrm{CaCl}_{2}$ plus 1 or $2 \% \mathrm{H}_{2} \mathrm{O}_{2}$ were superior in constricting the changing rate of skin color and decreasing rate of brightness. Thus, these applications showed the heighest values of hue angle and lightness as compared to the other treated and untreated fruits (control).

Treated 'Etmany' guavas by $4 \% \mathrm{CaCl}_{2}$ in combination with $2 \% \quad \mathrm{H}_{2} \mathrm{O}_{2}$ showed maximum values of hue angle (100.05 \& 94.79) and lightness (79.62 \& 80.67). On the other hand, untreated fruits (control) recorded the minimum values of hue angle (89.07 \& 84.24) and lightness (70.56 \& 70.39) in the first and second seasons, respectively.

The interaction effect between pre-storage treatments and storage periods showed significant difference interactions at $p \leq 0.05$ for hue angle and lightness of 'Etmany' guava fruits during the two seasons in this research.

The higher hue angle and lightness values of 'Etmany' guavas treated with $\mathrm{CaCl}_{2}, \mathrm{H}_{2} \mathrm{O}_{2}$ and their combinations especially treated fruits with $4 \% \mathrm{CaCl}_{2}$ plus 1 or $2 \% \mathrm{H}_{2} \mathrm{O}_{2}$ as compared to untreated fruits (control) might be due to the slower change of colour (Jain et al., 2003). In addition, a decrease of hue angle value with the advancement of storage period is attributed to the disappearance of the green color in fruits and appearance of yellow color (Dhillon \& Kaur, 2013). 
Table (6): Color represented as hue angle of 'Etmany' guava fruits as influenced by calcium chloride and hydrogen peroxide applications during storage time at $8 \pm 1^{\circ} \mathrm{C}$ plus two days shelf life at $18-23^{\circ} \mathrm{C}$

\begin{tabular}{|c|c|c|c|c|c|}
\hline \multirow{3}{*}{ Pre-storage applications } & \multicolumn{5}{|c|}{ Storage period (days) } \\
\hline & $\mathbf{0}$ & 7 & 14 & 21 & Means \\
\hline & \multicolumn{5}{|c|}{ Season 2015} \\
\hline Distilled water (control) & 107.41 b & 89.14 g-j & 79.15 o & 70.91 p & $86.65 \mathrm{~F}$ \\
\hline $2 \%$ calcium chloride $\left(\mathrm{CaCl}_{2}\right)$ & 109.25 ab & 91.84 e-h & 82.92 k-o & 80.12 no & 91.03 DE \\
\hline $4 \% \mathrm{CaCl}_{2}$ & 110.54 ab & 93.17 efg & 86.44 ijk & 81.68 l-o & $92.96 \mathrm{CD}$ \\
\hline $1 \%$ hydrogen peroxide $\left(\mathrm{H}_{2} \mathrm{O}_{2}\right)$ & 109.21 ab & 90.13 ghi & $81.43 \quad$ l-o & $78.95 \quad$ o & 89.93 E \\
\hline $2 \% \mathrm{H}_{2} \mathrm{O}_{2}$ & $109.61 \mathrm{ab}$ & 92.06 efg & 84.60 j-n & 80.46 mno & $91.68 \mathrm{DE}$ \\
\hline $2 \% \mathrm{CaCl}_{2}+1 \% \mathrm{H}_{2} \mathrm{O}_{2}$ & 111.09 ab & 96.32 cde & 87.25 h-k & 84.93 j-m & $94.90 \mathrm{BC}$ \\
\hline $2 \% \mathrm{CaCl}_{2}+2 \% \mathrm{H}_{2} \mathrm{O}_{2}$ & 111.49 ab & $97.62 \mathrm{~cd}$ & 89.85 ghi & $85.83 \quad$ i-l & $96.20 \mathrm{~B}$ \\
\hline $4 \% \mathrm{CaCl}_{2}+1 \% \mathrm{H}_{2} \mathrm{O}_{2}$ & $112.30 \mathrm{a}$ & 99.88 c & 92.84 efg & $88.64 \quad$ g-j & 98.41 A \\
\hline $4 \% \mathrm{CaCl}_{2}+2 \% \mathrm{H}_{2} \mathrm{O}_{2}$ & 113.66 a & $100.40 \mathrm{c}$ & 94.98 def & 91.14 fgh & $100.05 \mathrm{~A}$ \\
\hline \multirow[t]{2}{*}{ Means } & $110.51 \mathrm{~A}$ & 94.51 B & $86.61 \quad \mathrm{C}$ & 82.52 D & \\
\hline & \multicolumn{5}{|c|}{ Season 2016} \\
\hline Distilled water (control) & 101.77 b & $84.46 \quad$ g-j & $75.00 \quad 0$ & $67.19 p$ & 82.10 F \\
\hline $2 \%$ calcium chloride $\left(\mathrm{CaCl}_{2}\right)$ & 103.52 ab & 87.02 e-h & $78.57 \quad$ k-o & 75.91 no & 86.25 DE \\
\hline $4 \% \mathrm{CaCl}_{2}$ & 104.74 ab & 88.28 efg & 81.90 ijk & $77.40 \quad 1-0$ & 88.08 CD \\
\hline $1 \%$ hydrogen peroxide $\left(\mathrm{H}_{2} \mathrm{O}_{2}\right)$ & 103.48 ab & 85.40 ghi & $77.16 \quad$ l-o & $74.80 \quad$ o & $\begin{array}{|ll|}85.21 & \text { E } \\
\end{array}$ \\
\hline $2 \% \mathrm{H}_{2} \mathrm{O}_{2}$ & 103.86 ab & 87.23 efg & 80.15 j-n & 76.24 mno & 86.87 DE \\
\hline $2 \% \mathrm{CaCl}_{2}+1 \% \mathrm{H}_{2} \mathrm{O}_{2}$ & 105.26 ab & 91.26 cde & 82.67 h-k & $80.47 \quad$ j-m & 89.91 BC \\
\hline $2 \% \mathrm{CaCl}_{2}+2 \% \mathrm{H}_{2} \mathrm{O}_{2}$ & $105.64 \mathrm{ab}$ & $92.50 \quad c d$ & 85.13 ghi & 81.33 i-l & $91.15 \mathrm{~B}$ \\
\hline $4 \% \mathrm{CaCl}_{2}+1 \% \mathrm{H}_{2} \mathrm{O}_{2}$ & 106.40 a & $94.63 \mathrm{c}$ & 87.96 efg & 83.99 g-j & $93.25 \mathrm{~A}$ \\
\hline $4 \% \mathrm{CaCl}_{2}+2 \% \mathrm{H}_{2} \mathrm{O}_{2}$ & 107.69 a & 95.13 c & 90.00 def & 86.35 fgh & $94.79 \mathrm{~A}$ \\
\hline Means & 104.70 A & 89.55 B & $82.06 \mathrm{C}$ & 78.19 D & \\
\hline
\end{tabular}

Means followed by the same letters within pre-storage applications, storage periods and their interactions in each season are not significantly different at level $P \leq 0.05$ according to DMRT.

Table (7): Lightness of 'Etmany' guava fruits as influenced by calcium chloride and hydrogen peroxide applications during storage time at $8 \pm 1^{\circ} \mathrm{C}$ plus two days shelf life at $18-23^{\circ} \mathrm{C}$

\begin{tabular}{|c|c|c|c|c|c|c|c|}
\hline \multirow{3}{*}{ Pre-storage applications } & \multicolumn{7}{|c|}{ Storage period (days) } \\
\hline & $\mathbf{0}$ & 7 & 14 & \multicolumn{2}{|c|}{21} & \multicolumn{2}{|c|}{ Means } \\
\hline & \multicolumn{7}{|c|}{ Season 2015} \\
\hline Distilled water (control) & 84.03 a-e & 76.40 g-k & 66.80 & 60.17 & $\mathbf{0}$ & 71.85 & $\mathbf{F}$ \\
\hline $2 \%$ calcium chloride $\left(\mathrm{CaCl}_{2}\right)$ & 85.44 a-d & 77.49 & 71.09 & 66.76 & n & 75.20 & $\mathbf{E}$ \\
\hline $4 \% \mathrm{CaCl}_{2}$ & $85.67 \quad$ a-d & 79.95 c-h & 74.59 & 69.89 & Imn & $\mathbf{7 7 . 5 3}$ & CDE \\
\hline $1 \%$ hydrogen peroxide $\left(\mathrm{H}_{2} \mathrm{O}_{2}\right)$ & 85.41 a-d & 77.12 & 71.26 & 66.96 & $\mathbf{n}$ & 75.19 & $\mathbf{E}$ \\
\hline $2 \% \mathrm{H}_{2} \mathrm{O}_{2}$ & 85.56 a-d & 78.88 & 73.34 & 68.54 & mn & 76.58 & $\mathbf{D E}$ \\
\hline $2 \% \mathrm{CaCl}_{2}+1 \% \mathrm{H}_{2} \mathrm{O}_{2}$ & 85.83 abc & $80.26 \quad$ b-h & 76.52 & 72.56 & $\mathbf{j}$-m & 78.79 & BCD \\
\hline $2 \% \mathrm{CaCl}_{2}+2 \% \mathrm{H}_{2} \mathrm{O}_{2}$ & 85.90 ab & 80.73 & 77.40 & 73.28 & i-m & 79.33 & BC \\
\hline $4 \% \mathrm{CaCl}_{2}+1 \% \mathrm{H}_{2} \mathrm{O}_{2}$ & 86.02 & 82.76 & 79.81 & 76.62 & g-k & 81.30 & $\mathbf{A B}$ \\
\hline $4 \% \mathrm{CaCl}_{2}+2 \% \mathrm{H}_{2} \mathrm{O}_{2}$ & 86.30 & 84.21 & 81.65 & 78.50 & e-i & 82.67 & $\mathbf{A}$ \\
\hline \multirow[t]{2}{*}{ Means } & 85.57 & 79.76 & 74.72 & 70.36 & D & & \\
\hline & \multicolumn{7}{|c|}{ Season 2016} \\
\hline Distilled water (c & 86.19 a-e & $78.36 \mathrm{~g}-\mathrm{k}$ & $68.51 \mathrm{n}$ & 61.72 & $\mathbf{0}$ & 73.70 & $\mathbf{F}$ \\
\hline $2 \%$ calcium chloride $\left(\mathrm{CaCl}_{2}\right)$ & 87.63 a-d & $79.48 \mathrm{f}-\mathrm{j}$ & 72.92 k-n & 68.47 & n & 77.13 & $\mathbf{E}$ \\
\hline $4 \% \mathrm{CaCl}_{2}$ & 87.87 a-d & 82.00 c-h & 76.50 h-l & 71.69 & $\operatorname{lmn}$ & 79.52 & CDE \\
\hline $1 \%$ hydrogen peroxide $\left(\mathrm{H}_{2} \mathrm{O}_{2}\right)$ & 87.60 a-d & $79.10 \mathrm{f}-\mathrm{j}$ & $73.09 \quad k-n$ & 68.68 & n & 77.12 & $\mathbf{E}$ \\
\hline $2 \% \mathrm{H}_{2} \mathrm{O}_{2}$ & 87.75 a-d & 80.90 e-i & 75.23 i-m & 70.30 & mn & 78.55 & DE \\
\hline $2 \% \mathrm{CaCl}_{2}+1 \% \mathrm{H}_{2} \mathrm{O}_{2}$ & 88.04 abc & 82.33 b-h & 78.49 g-k & 74.42 & j-m & 80.82 & BCD \\
\hline $2 \% \mathrm{CaCl}_{2}+2 \% \mathrm{H}_{2} \mathrm{O}_{2}$ & 88.10 ab & 82.80 a-g & $79.39 \quad f-j$ & 75.16 & i-m & 81.36 & BC \\
\hline $4 \% \mathrm{CaCl}_{2}+1 \% \mathrm{H}_{2} \mathrm{O}_{2}$ & 88.23 ab & 84.88 a-f & 81.86 d-h & 78.59 & g-k & 83.39 & $\mathbf{A B}$ \\
\hline $4 \% \mathrm{CaCl}_{2}+2 \% \mathrm{H}_{2} \mathrm{O}_{2}$ & 88.52 a & 86.38 a-e & 83.75 a-g & 80.52 & e-i & 84.79 & $\mathbf{A}$ \\
\hline Means & $87.77 \mathrm{~A}$ & 81.80 B & $76.64 \mathrm{C}$ & 72.17 & D & & \\
\hline
\end{tabular}

Means followed by the same letters within pre-storage applications, storage periods and their interactions in each season are not significantly different at level $P \leq 0.05$ according to DMRT. 
Moreover, a decrease in lightness value with the prolonging of storage period is due to fruit senescence (Mohamed et al., 2016).

The results in the present study are in agreement with findings of Shaaban (2006) who reported that, hue angle and lightness values increased by treating guavas with $\mathrm{CaCl}_{2}$ as compared to untreated fruits during storage and these values decreased with the advancement of storage period. Moreover, the application of $1 \%$ $\mathrm{CaCl}_{2}$ was efficient in delaying loss of skin color of guavas during shelf life for 12 days (Kumar et al., 2012). Furthermore, these findings are in accordance with the findings of Dhillon \& Kaur (2013) on mango fruits where, $\mathrm{CaCl}_{2}$ treatment showed a beneficial effect in constricted the variability in color than the control treatment. In addition, postharvest application of $2 \% \mathrm{H}_{2} \mathrm{O}_{2}$ delayed skin color development of stored 'Valencia' oranges by the increase of hue angle value and also reduced the deterioration of lightness value as compared to the untreated fruits (Mohamed et al., 2016).

\subsection{Chemical quality attributes as affected by} pre-storage applications of calcium chloride, hydrogen peroxide and their combinations of 'Etmany' guava fruits during storage

\subsubsection{Fruit content of vitamin $C$}

The data in Table (8) illustrated that, a decline trend in vitamin $\mathrm{C}$ content was observed with the advancement of cold storage period at $8^{\circ} \mathrm{C}$ plus two days shelf life at ambient temperature $\left(18-23^{\circ} \mathrm{C}\right)$ in both seasons. Our results revealed that, minimum vitamin $\mathrm{C}$ content of 'Etmany' guavas was observed at the end of storage period and the values reached of $76.30 \& 77.96 \mathrm{mg} / 100 \mathrm{ml}$ juice in the first and second seasons, respectively.

The data in Table (8) clearly revealed that, all pre-storage applications significantly reduced the deterioration rate in vitamin $\mathrm{C}$ content as compared to the untreated fruits (control) during the cold storage period at $8^{\circ} \mathrm{C}$ followed by two days shelf life at ambient temperature $\left(18-23^{\circ} \mathrm{C}\right)$ in both seasons.

Moreover, individual treatments of $\mathrm{CaCl}_{2}$ were more effective in maintaining vitamin $\mathrm{C}$ content as compared to individual treatments of $\mathrm{H}_{2} \mathrm{O}_{2}$ in both seasons. In addition, 'Etmany' guava fruits treated with the combination treatments especially those treated with $4 \%$ $\mathrm{CaCl}_{2}$ plus 1 or $2 \% \mathrm{H}_{2} \mathrm{O}_{2}$ had significantly the highest vitamin $\mathrm{C}$ content in the two seasons. Moreover, the loss of vitamin $\mathrm{C}$ content was more rapid in the untreated fruits (control) during the two seasons under this investigation.

The data also indicated that, dipping 'Etmany' winter guavas in $4 \% \mathrm{CaCl}_{2}$ plus $2 \%$ $\mathrm{H}_{2} \mathrm{O}_{2}$ treatment had the highest vitamin $\mathrm{C}$ content (102.14 \& $103.76 \mathrm{mg} / 100 \mathrm{ml}$ juice) in 2015 and 2016 seasons, respectively. On the contrast, the untreated fruits (control) recorded the lowest vitamin $\mathrm{C}$ content $(82.30 \& 83.91$ $\mathrm{mg} / 100 \mathrm{ml}$ juice) in the first and the second seasons, respectively.

The data also declared that, there was a significant interaction among pre-storage treatments and storage periods at $p \leq 0.05$ for vitamin $\mathrm{C}$ content of 'Etmany' guava fruits in both seasons in the current investigation.

Higher vitamin $\mathrm{C}$ content in 'Etmany' guavas treated with $\mathrm{CaCl}_{2}, \mathrm{H}_{2} \mathrm{O}_{2}$ and their combinations especially those treated with $4 \% \mathrm{CaCl}_{2}$ in combination with 1 or $2 \% \mathrm{H}_{2} \mathrm{O}_{2}$ as compared to the untreated fruits might be ascribed to the levitation in the activity of antioxidant enzymes as documented by Khandaker et al. (2012) and Reena (2016). Thus, these treatments retarded the oxidation of vitamin $\mathrm{C}$ by slowing down the rate of conversion of ascorbic acid into dehydro ascorbic acid (Davey et al., 2000). In addition, the loss of guavas content in ascorbic acid during storage period might be due to a rapid conversion of L-ascorbic acid into dehydroascorbic acid in the presence of oxidizing enzymes like ascorbic acid oxidase and ascorbate peroxidase (Davey et al., 2000).

These results are in agreement with those obtained by Hiwale \& Singh (2003), Shaaban (2006), Mahajan et al. (2011), Phani et al. (2016), Dohre (2016) and Reena (2016). They all claimed that, guava fruits treated with $\mathrm{CaCl}_{2}$ had higher vitamin $\mathrm{C}$ content than the untreated fruits and also they demonstrated that vitamin $\mathrm{C}$ decreased with the elongation of storage period. In addition, the obtained results were similar to those mentioned by Ismail et al. (2010) who stated that, immersing 'Maamoura' guava fruits in $2 \% \mathrm{CaCl}_{2}$ or $2 \% \mathrm{H}_{2} \mathrm{O}_{2}$ for four minutes plus fumigation with lemon grass oil treatment reduced the rate of Vitamin $\mathrm{C}$ degradation during cold storage at $8^{\circ} \mathrm{C}$ for 15 days as compared to the control treatment. In addition, these results are in harmony with the findings of Mohamed et al. (2016) who reported that immersing 'Valencia' oranges in $2 \% \mathrm{H}_{2} \mathrm{O}_{2}$ for five minutes reduced the deterioration of vitamin $\mathrm{C}$ as compared to the untreated fruits during cold storage at $8^{\circ} \mathrm{C}$ for fifteen weeks. 
Table (8): Vitamin C content (mg/100 ml juice) of 'Etmany' guava fruits as influenced by calcium chloride and hydrogen peroxide applications during storage time at $8 \pm 1^{\circ} \mathrm{C}$ plus two days shelf life at $18-23^{\circ} \mathrm{C}$

\begin{tabular}{|c|c|c|c|c|c|}
\hline \multirow{3}{*}{ Pre-storage applications } & \multicolumn{5}{|c|}{ Storage period (days) } \\
\hline & $\mathbf{0}$ & 7 & 14 & 21 & Means \\
\hline & \multicolumn{5}{|c|}{ Season 2015} \\
\hline Distilled water (control) & 110.77 ab & 84.85 i-l & $71.90 \mathrm{mn}$ & $61.67 \quad 0$ & 82.30 G \\
\hline $2 \%$ calcium chloride $\left(\mathrm{CaCl}_{2}\right)$ & 112.31 a & $92.12 \mathrm{f}-\mathrm{i}$ & 81.90 kl & $72.78 \mathrm{~m}$ & 89.78 DE \\
\hline $4 \% \mathrm{CaCl}_{2}$ & 113.85 a & 94.55 efg & 85.24 i-l & $75.00 \mathrm{~m}$ & 92.16 D \\
\hline $1 \%$ hydrogen peroxide $\left(\mathrm{H}_{2} \mathrm{O}_{2}\right)$ & 111.79 ab & 87.27 h-k & $74.76 \mathrm{~m}$ & $65.00 \quad 0$ & 84.71 FG \\
\hline $2 \% \mathrm{H}_{2} \mathrm{O}_{2}$ & 112.82 a & 93.33 e-h & $78.57 \quad \mathrm{~lm}$ & 66.11 no & 87.71 EF \\
\hline $2 \% \mathrm{CaCl}_{2}+1 \% \mathrm{H}_{2} \mathrm{O}_{2}$ & 114.36 a & 97.58 def & $89.52 \quad \mathrm{~g}-\mathrm{j}$ & 83.33 jkl & $96.20 \mathrm{C}$ \\
\hline $2 \% \mathrm{CaCl}_{2}+2 \% \mathrm{H}_{2} \mathrm{O}_{2}$ & 114.87 a & 100.00 cde & 91.90 f-i & 85.00 i-l & 97.94 BC \\
\hline $4 \% \mathrm{CaCl}_{2}+1 \% \mathrm{H}_{2} \mathrm{O}_{2}$ & 115.38 a & $103.03 \mathrm{~cd}$ & 93.81 e-h & 87.22 h-k & $99.86 \mathrm{AB}$ \\
\hline $4 \% \mathrm{CaCl}_{2}+2 \% \mathrm{H}_{2} \mathrm{O}_{2}$ & 115.90 a & 105.45 bc & 96.67 d-g & $90.56 \mathrm{f}-\mathrm{j}$ & 102.14 A \\
\hline \multirow[t]{2}{*}{ Means } & $113.56 \mathrm{~A}$ & $95.35 \mathrm{~B}$ & $84.92 \mathrm{C}$ & $76.30 \quad \mathrm{D}$ & \\
\hline & \multicolumn{5}{|c|}{ Season 2016} \\
\hline Distilled water (control) & 112.31 ab & $86.67 \mathrm{j}-\mathrm{m}$ & 73.33 op & $63.33 q$ & 83.91 F \\
\hline $2 \%$ calcium chloride $\left(\mathrm{CaCl}_{2}\right)$ & 113.85 a & $93.94 \mathrm{f}-\mathrm{i}$ & $83.33 \quad \operatorname{lm}$ & 74.44 no & 91.39 D \\
\hline $4 \% \mathrm{CaCl}_{2}$ & 115.38 a & 96.36 e-h & 86.67 j-m & 76.67 no & 93.77 D \\
\hline $1 \%$ hydrogen peroxide $\left(\mathrm{H}_{2} \mathrm{O}_{2}\right)$ & 113.33 ab & 89.09 i-l & 76.19 no & $66.67 \quad q$ & 86.32 EF \\
\hline $2 \% \mathrm{H}_{2} \mathrm{O}_{2}$ & 114.36 a & $91.52 \mathrm{~g}-\mathrm{k}$ & $80.00 \mathrm{mn}$ & $67.78 \mathrm{pq}$ & 88.41 E \\
\hline $2 \% \mathrm{CaCl}_{2}+1 \% \mathrm{H}_{2} \mathrm{O}_{2}$ & 115.90 a & 99.39 def & 90.95 h-k & 85.00 klm & 97.81 C \\
\hline $2 \% \mathrm{CaCl}_{2}+2 \% \mathrm{H}_{2} \mathrm{O}_{2}$ & 116.41 a & 101.82 cde & 93.33 f-j & $86.67 \quad j-m$ & $99.56 \mathrm{BC}$ \\
\hline $4 \% \mathrm{CaCl}_{2}+1 \% \mathrm{H}_{2} \mathrm{O}_{2}$ & 116.92 a & $104.85 \mathrm{~cd}$ & 95.24 e-i & 88.89 i-l & 101.47 AB \\
\hline $4 \% \mathrm{CaCl}_{2}+2 \% \mathrm{H}_{2} \mathrm{O}_{2}$ & 117.44 a & 107.27 bc & 98.10 efg & 92.22 g-j & 103.76 A \\
\hline Means & $115.10 \mathrm{~A}$ & 96.77 B & $86.35 \mathrm{C}$ & 77.96 D & \\
\hline
\end{tabular}

Means followed by the same letters within pre-storage applications, storage periods and their interactions in each season are not significantly different at level $P \leq 0.05$ according to DMRT.

\subsubsection{Fruit contents of total soluble solids (TSS),} titratable acidity (TA) and TSS/TA ratio

The data in Table (9) demonstrate that, the maximum 'Etmany' guavas content of TSS was recorded at 14 days of cold storage period plus two days shelf life at ambient temperature (12.09 \& $12.17{ }^{\circ}$ Brix) followed by a significant decrease at the end of the experiment $(11.96 \&$ $12.04{ }^{\circ}$ Brix) in 2015 and 2016 seasons, respectively. On the other side, declining trend of guava fruits content in TA was observed with prolonging of storage period in both seasons and the minimum guavas content of TA was observed at the end of storage period $(0.64 \&$ $0.58 \mathrm{~g}$ citric acid/100 $\mathrm{ml}$ juice) in the first and second seasons, respectively (Table 10). While, rising trend of TSS/TA ratio was recorded with the advancement of storage period in the two seasons and the maximum TSS/TA ratio of guava fruits was recorded at the end of the storage period (19.62 \& 22.27) in 2015 and 2016 seasons, respectively (Table 11).

Statistical data in Table (9) showed that, immersing 'Etmany' guavas in 2 or $4 \% \mathrm{CaCl}_{2}$ in combination with 1 or $2 \% \mathrm{H}_{2} \mathrm{O}_{2}$ treatments slowed the increase of TSS content than the individual and the control treatments during cold storage period at $8^{\circ} \mathrm{C}$ followed by two days shelf life at ambient temperature $\left(18-23^{\circ} \mathrm{C}\right)$ in both seasons. In the control treatment, fruit content of TSS was increased till seven days of the storage period, while the peak of TSS was recorded a week later by individual treatments. After that, a decrease trend in the control and the individual treatments was noticed till the end of the storage period in the two seasons under this investigation. On the contrary, continuaus increase of fruit content in TSS was noticed in combined treatments until end of the experiment.

Moreover, the data in Tables (10 and 11) revealed that, all pre-storage treatments of $\mathrm{CaCl}_{2}, \mathrm{H}_{2} \mathrm{O}_{2}$ and their combinations of 'Etmany' guava fruits significantly maintained fruit content in TA as compared to the control in both seasons. In addition, these applications slowed the increase of TSS/TA ratio than the untreated fruits (control). Our results indicated that, treated 'Etmany' guava fruits with combined treatments especially those treated with $4 \% \mathrm{CaCl}_{2}$ in combination with $\mathrm{H}_{2} \mathrm{O}_{2}$ at 1 or $2 \%$ had significantly the lowest fruit contents of TSS and TSS/TA ratio, on contrary had significantly the highest fruit content of TA in the two seasons.

The data in Tables (9, 10 and 11) also emerged that, soaking 'Etmany' guavas in 4\% $\mathrm{CaCl}_{2}$ plus $2 \% \mathrm{H}_{2} \mathrm{O}_{2}$ treatment had the lowest values of TSS (10.03 \& $10.12{ }^{\circ}$ Brix) and TSS/TA ratio (11.02\& 11.96) in 2015 and 2016 
Table (9): Total soluble solids contents ( $\left.{ }^{\circ} \mathrm{Brix}\right)$ of 'Etmany' guava fruits as influenced by calcium chloride and hydrogen peroxide applications during storage time at $8 \pm 1^{\circ} \mathrm{C}$ plus two days shelf life at $18-23^{\circ} \mathrm{C}$

\begin{tabular}{|c|c|c|c|c|c|c|c|c|}
\hline \multirow{3}{*}{ Pre-storage applications } & \multicolumn{8}{|c|}{ Storage period (days) } \\
\hline & \multicolumn{2}{|c|}{$\mathbf{0}$} & 7 & \multirow{2}{*}{\begin{tabular}{|c|}
14 \\
Season 2015
\end{tabular}} & \multicolumn{2}{|c|}{21} & \multicolumn{2}{|c|}{ Means } \\
\hline & & & & & & & & \\
\hline Distilled water (control) & 8.53 & no & $12.40 \mathrm{ab}$ & 11.93 c-g & $\mathbf{1 1 . 4 7}$ & h-k & 11.08 & $\mathbf{A}$ \\
\hline $2 \%$ calcium chloride $\left(\mathrm{CaCl}_{2}\right)$ & 8.40 & n-q & 11.40 ijk & $12.40 \mathrm{ab}$ & $\mathbf{1 1 . 8 0}$ & e-i & 11.00 & $\mathbf{A}$ \\
\hline $4 \% \mathrm{CaCl}_{2}$ & 8.27 & opq & $11.07 \mathrm{k}$ & $12.27 \quad$ a-d & 12.00 & b-f & 10.90 & $\mathbf{A}$ \\
\hline $1 \%$ hydrogen peroxide $\left(\mathrm{H}_{2} \mathrm{O}_{2}\right)$ & 8.47 & nop & $11.53 \mathrm{~g}-\mathrm{j}$ & 12.47 a & $\mathbf{1 1 . 6 7}$ & f-i & 11.03 & $\mathbf{A}$ \\
\hline $2 \% \mathrm{H}_{2} \mathrm{O}_{2}$ & 8.33 & n-q & $11.20 \mathrm{jk}$ & 12.33 abc & $\mathbf{1 1 . 8 7}$ & d-h & 10.93 & $\mathbf{A}$ \\
\hline $2 \% \mathrm{CaCl}_{2}+1 \% \mathrm{H}_{2} \mathrm{O}_{2}$ & 8.20 & opq & $9.20 \quad 1$ & 12.13 a-e & $\mathbf{1 2 . 4 7}$ & $\mathbf{a}$ & 10.50 & $\mathbf{B}$ \\
\hline $2 \% \mathrm{CaCl}_{2}+2 \% \mathrm{H}_{2} \mathrm{O}_{2}$ & 8.13 & opq & $9.13 \mathrm{~lm}$ & $11.93 \mathrm{c}-\mathrm{g}$ & 12.27 & a-d & 10.37 & $\mathbf{B C}$ \\
\hline $4 \% \mathrm{CaCl}_{2}+1 \% \mathrm{H}_{2} \mathrm{O}_{2}$ & 8.07 & pq & $9.00 \mathrm{~lm}$ & 11.80 e-i & 12.20 & a-e & 10.27 & $\mathbf{C}$ \\
\hline $4 \% \mathrm{CaCl}_{2}+2 \% \mathrm{H}_{2} \mathrm{O}_{2}$ & 8.00 & q & $8.73 \mathrm{mn}$ & 11.53 g-j & $\mathbf{1 1 . 8 7}$ & d-h & $\mathbf{1 0 . 0 3}$ & D \\
\hline \multirow[t]{2}{*}{ Means } & 8.27 & D & $10.41 \mathrm{C}$ & $12.09 \mathrm{~A}$ & 11.96 & B & & \\
\hline & \multicolumn{8}{|c|}{ Season 2016} \\
\hline Distilled water (control) & 8.67 & no & 12.60 a & 12.27 a-d & $\mathbf{1 1 . 5 3}$ & g-k & 11.27 & $\mathbf{A}$ \\
\hline $2 \%$ calcium chloride $\left(\mathrm{CaCl}_{2}\right)$ & 8.53 & opq & 11.47 h-k & $12.53 \mathrm{ab}$ & 12.00 & def & 11.13 & $\mathbf{A B}$ \\
\hline $4 \% \mathrm{CaCl}_{2}$ & 8.40 & opq & $11.13 \mathrm{k}$ & 12.47 abc & 12.20 & a-d & 11.05 & B \\
\hline $1 \%$ hydrogen peroxide $\left(\mathrm{H}_{2} \mathrm{O}_{2}\right)$ & 8.60 & op & $11.60 \mathrm{f}-\mathrm{j}$ & 12.60 a & $\mathbf{1 1 . 8 7}$ & d-h & 11.17 & $\mathbf{A B}$ \\
\hline $2 \% \mathrm{H}_{2} \mathrm{O}_{2}$ & 8.47 & opq & 11.27 jk & 12.47 abc & 12.07 & cde & 11.07 & $\mathbf{A B}$ \\
\hline $2 \% \mathrm{CaCl}_{2}+1 \% \mathrm{H}_{2} \mathrm{O}_{2}$ & 8.33 & opq & $9.47 \quad 1$ & 12.13 b-e & 12.47 & abc & 10.60 & $\mathbf{C}$ \\
\hline $2 \% \mathrm{CaCl}_{2}+2 \% \mathrm{H}_{2} \mathrm{O}_{2}$ & 8.27 & opq & $9.40 \quad 1$ & 11.93 d-g & 12.20 & a-d & 10.45 & CD \\
\hline $4 \% \mathrm{CaCl}_{2}+1 \% \mathrm{H}_{2} \mathrm{O}_{2}$ & 8.20 & pq & $9.27 \quad \operatorname{lm}$ & 11.73 e-i & 12.07 & cde & 10.32 & D \\
\hline $4 \% \mathrm{CaCl}_{2}+2 \% \mathrm{H}_{2} \mathrm{O}_{2}$ & 8.13 & $\mathbf{q}$ & 9.00 & 11.40 ijk & 11.93 & d-g & 10.12 & $\mathbf{E}$ \\
\hline Means & 8.40 & D & $10.58 \quad \mathrm{C}$ & $12.17 \mathrm{~A}$ & $\mathbf{1 2 . 0 4}$ & $\mathbf{B}$ & & \\
\hline
\end{tabular}

Means followed by the same letters within pre-storage applications, storage periods and their interactions in each season are not significantly different at level $\mathrm{P} \leq 0.05$ according to DMRT.

Table (10): Titratable acidity (g citric acid/100 ml juice) of 'Etmany' guava fruits as influenced by calcium chloride and hydrogen peroxide applications during storage time at $8 \pm 1^{\circ} \mathrm{C}$ plus two days shelf life at $18-23^{\circ} \mathrm{C}$.

\begin{tabular}{|c|c|c|c|c|c|c|c|c|c|c|}
\hline \multirow{3}{*}{ Pre-storage applications } & \multicolumn{10}{|c|}{ Storage period (days) } \\
\hline & \multicolumn{2}{|c|}{$\mathbf{0}$} & \multirow{2}{*}{\multicolumn{2}{|c|}{7}} & \multirow{2}{*}{\multicolumn{2}{|c|}{14}} & \multicolumn{2}{|c|}{21} & \multicolumn{2}{|c|}{ Means } \\
\hline & \multicolumn{6}{|c|}{ Season 2015} & & & & \\
\hline Distilled water (control) & 0.82 & g-k & $\mathbf{0 . 6 5}$ & o-r & 0.57 & rst & 0.47 & $\mathbf{u}$ & 0.63 & $\mathbf{G}$ \\
\hline $2 \%$ calcium chloride $\left(\mathrm{CaCl}_{2}\right)$ & 0.87 & $c-j$ & $\mathbf{0 . 7 6}$ & $\mathbf{j}-\mathbf{n}$ & 0.66 & n-r & $\mathbf{0 . 5 3}$ & stu & $\mathbf{0 . 7 0}$ & $\mathbf{E F}$ \\
\hline $4 \% \mathrm{CaCl}_{2}$ & 0.91 & b-h & $\mathbf{0 . 8 0}$ & h-k & 0.70 & l-p & 0.62 & p-s & 0.76 & D \\
\hline 1\% hydrogen peroxide $\left(\mathrm{H}_{2} \mathrm{O}_{2}\right)$ & 0.84 & e-j & 0.69 & l-q & 0.62 & p-s & 0.51 & tu & 0.66 & FG \\
\hline $2 \% \mathrm{H}_{2} \mathrm{O}_{2}$ & 0.89 & b-i & $\mathbf{0 . 7 8}$ & i-m & 0.68 & m-q & 0.59 & q-t & 0.74 & DE \\
\hline $2 \% \mathrm{CaCl}_{2}+1 \% \mathrm{H}_{2} \mathrm{O}_{2}$ & 0.93 & a-f & 0.85 & d-j & 0.76 & $\mathbf{j}-\mathbf{m}$ & 0.70 & l-p & 0.81 & $\mathbf{C}$ \\
\hline $2 \% \mathrm{CaCl}_{2}+2 \% \mathrm{H}_{2} \mathrm{O}_{2}$ & 0.95 & a-d & $\mathbf{0 . 8 9}$ & b-h & 0.83 & $\mathbf{f}-\mathbf{j}$ & 0.72 & k-o & 0.85 & BC \\
\hline $4 \% \mathrm{CaCl}_{2}+1 \% \mathrm{H}_{2} \mathrm{O}_{2}$ & 0.98 & $\mathbf{a b}$ & 0.94 & a-e & 0.87 & c-i & 0.79 & i-l & 0.89 & $\mathbf{A B}$ \\
\hline $4 \% \mathrm{CaCl}_{2}+2 \% \mathrm{H}_{2} \mathrm{O}_{2}$ & 1.02 & $\mathbf{a}$ & 0.96 & abc & 0.91 & b-g & 0.83 & $\mathbf{f - j}$ & 0.93 & $\mathbf{A}$ \\
\hline \multirow[t]{2}{*}{ Means } & 0.91 & $\mathbf{A}$ & $\mathbf{0 . 8 1}$ & $\mathbf{B}$ & 0.73 & $\mathbf{C}$ & 0.64 & D & & \\
\hline & \multicolumn{10}{|c|}{ Season 2016} \\
\hline Distilled water (control) & 0.75 & h-m & $\mathbf{0 . 5 8}$ & $\mathbf{r}-\mathbf{u}$ & 0.51 & uvw & 0.40 & $\mathbf{x}$ & 0.56 & \\
\hline $2 \%$ calcium chloride $\left(\mathrm{CaCl}_{2}\right)$ & 0.80 & e-k & 0.69 & m-q & 0.59 & rst & 0.47 & vwx & 0.64 & $\mathbf{F}$ \\
\hline $4 \% \mathrm{CaCl}_{2}$ & 0.84 & b-g & 0.74 & i-n & 0.64 & o-r & 0.55 & stu & 0.69 & $\mathbf{E}$ \\
\hline $1 \%$ hydrogen peroxide $\left(\mathrm{H}_{2} \mathrm{O}_{2}\right)$ & 0.78 & g-m & 0.63 & p-s & 0.55 & stu & 0.45 & $\mathbf{w x}$ & 0.60 & $\mathbf{G}$ \\
\hline $2 \% \mathrm{H}_{2} \mathrm{O}_{2}$ & 0.82 & c-i & 0.71 & k-o & 0.62 & qrs & $\mathbf{0 . 5 3}$ & tuv & 0.67 & $\mathbf{E F}$ \\
\hline $2 \% \mathrm{CaCl}_{2}+1 \% \mathrm{H}_{2} \mathrm{O}_{2}$ & 0.87 & b-f & $\mathbf{0 . 7 8}$ & f-l & 0.70 & l-p & 0.64 & o-r & 0.75 & D \\
\hline $2 \% \mathrm{CaCl}_{2}+2 \% \mathrm{H}_{2} \mathrm{O}_{2}$ & 0.89 & a-d & $\mathbf{0 . 8 3}$ & b-h & 0.76 & g-m & 0.66 & n-r & 0.78 & $\mathbf{C}$ \\
\hline $4 \% \mathrm{CaCl}_{2}+1 \% \mathrm{H}_{2} \mathrm{O}_{2}$ & 0.91 & $\mathbf{a b}$ & 0.87 & b-e & 0.81 & d-j & 0.72 & j-n & $\mathbf{0 . 8 3}$ & B \\
\hline $4 \% \mathrm{CaCl}_{2}+2 \% \mathrm{H}_{2} \mathrm{O}_{2}$ & 0.95 & $\mathbf{a}$ & $\mathbf{0 . 8 9}$ & abc & $\mathbf{0 . 8 5}$ & b-g & 0.76 & g-m & 0.86 & $\mathbf{A}$ \\
\hline Means & 0.85 & $\mathbf{A}$ & 0.75 & B & 0.67 & $\mathbf{C}$ & 0.58 & D & & \\
\hline
\end{tabular}

Means followed by the same letters within pre-storage applications, storage periods and their interactions in each season are not significantly different at level $P \leq 0.05$ according to DMRT. 
Table (11): Total soluble solids/titratable acidity ratio of 'Etmany' guava fruits as influenced by calcium chloride and hydrogen peroxide applications during storage time at $8 \pm 1^{\circ} \mathrm{C}$ plus two days shelf life at $18-23^{\circ} \mathrm{C}$.

\begin{tabular}{|c|c|c|c|c|c|c|}
\hline \multirow{3}{*}{ Pre-storage applications } & \multicolumn{6}{|c|}{ Storage period (days) } \\
\hline & $\mathbf{0}$ & 7 & \multirow{2}{*}{\begin{tabular}{|c|}
14 \\
Season 201
\end{tabular}} & \multirow[t]{2}{*}{21} & \multicolumn{2}{|c|}{ Means } \\
\hline & & & & & & \\
\hline Distilled water (control) & 10.41 opq & 19.48 d-g & 21.00 bcd & 26.14 a & 19.26 & $\mathbf{A}$ \\
\hline $2 \%$ calcium chloride $\left(\mathrm{CaCl}_{2}\right)$ & $9.76 \mathrm{pq}$ & 15.03 k-n & $18.90 \mathrm{~d}-\mathrm{h}$ & 22.69 bc & 16.59 & $\mathbf{B C}$ \\
\hline $4 \% \mathrm{CaCl}_{2}$ & $9.10 \mathrm{pq}$ & $13.83 \mathrm{mn}$ & 17.52 f-k & $19.56 \mathrm{~d}-\mathrm{g}$ & 15.00 & D \\
\hline $1 \%$ hydrogen peroxide $\left(\mathrm{H}_{2} \mathrm{O}_{2}\right)$ & $10.06 \mathrm{pq}$ & 16.81 h-l & 20.31 cde & 23.19 b & 17.59 & B \\
\hline $2 \% \mathrm{H}_{2} \mathrm{O}_{2}$ & $9.41 \mathrm{pq}$ & $14.42 \operatorname{lmn}$ & $18.42 \mathrm{~d}-\mathrm{i}$ & 20.04 def & 15.57 & CD \\
\hline $2 \% \mathrm{CaCl}_{2}+1 \% \mathrm{H}_{2} \mathrm{O}_{2}$ & $8.80 \mathrm{pq}$ & 10.86 op & 15.89 i-m & 17.91 e-j & 13.36 & $\mathbf{E}$ \\
\hline $2 \% \mathrm{CaCl}_{2}+2 \% \mathrm{H}_{2} \mathrm{O}_{2}$ & $8.54 \mathrm{pq}$ & 10.23 opq & $14.49 \operatorname{lmn}$ & $17.03 \mathrm{~g}-\mathrm{I}$ & 12.57 & EF \\
\hline $4 \% \mathrm{CaCl}_{2}+1 \% \mathrm{H}_{2} \mathrm{O}_{2}$ & $8.27 \mathrm{pq}$ & $9.67 \mathrm{pq}$ & $13.58 \mathrm{mn}$ & $15.62 \mathrm{j}-\mathrm{m}$ & 11.79 & FG \\
\hline $4 \% \mathrm{CaCl}_{2}+2 \% \mathrm{H}_{2} \mathrm{O}_{2}$ & $\begin{array}{|ll|}7.89 & q \\
\end{array}$ & $9.15 \mathrm{pq}$ & \begin{tabular}{|l|}
12.65 no \\
\end{tabular} & $14.39 \operatorname{lmn}$ & 11.02 & $\mathbf{G}$ \\
\hline \multirow[t]{2}{*}{ Means } & $9.14 \mathrm{D}$ & 13.28 C & $16.97 \mathrm{~B}$ & $19.62 \mathrm{~A}$ & & \\
\hline & \multicolumn{6}{|c|}{ Season 2016} \\
\hline Distilled water (control) & $11.51 \mathrm{mno}$ & 22.18 cde & 24.41 bc & 31.05 a & 22.29 & $\mathbf{A}$ \\
\hline $2 \%$ calcium chloride $\left(\mathrm{CaCl}_{2}\right)$ & 10.74 mno & 16.59 hij & 21.15 def & 26.49 b & 18.74 & BC \\
\hline $4 \% \mathrm{CaCl}_{2}$ & 9.98 no & $15.20 \quad \mathbf{j k}$ & 19.59 e-h & 22.18 cde & 16.74 & D \\
\hline $1 \%$ hydrogen peroxide $\left(\mathrm{H}_{2} \mathrm{O}_{2}\right)$ & $11.10 \mathrm{mno}$ & 18.76 f-i & \begin{tabular}{|l|l|}
22.91 & cd \\
\end{tabular} & 27.05 b & 19.95 & $\mathbf{B}$ \\
\hline $2 \% \mathrm{H}_{2} \mathrm{O}_{2}$ & 10.33 no & $15.87 \quad$ ijk & 20.59 def & $22.84 \mathrm{~cd}$ & 17.41 & CD \\
\hline $2 \% \mathrm{CaCl}_{2}+1 \% \mathrm{H}_{2} \mathrm{O}_{2}$ & 9.63 no & 12.13 Imn & $17.33 \mathrm{~g}-\mathrm{j}$ & 19.72 efg & 14.70 & $\mathbf{E}$ \\
\hline $2 \% \mathrm{CaCl}_{2}+2 \% \mathrm{H}_{2} \mathrm{O}_{2}$ & 9.33 no & 11.40 mno & 15.69 ijk & $18.57 \mathrm{f}-\mathrm{i}$ & 13.75 & EF \\
\hline $4 \% \mathrm{CaCl}_{2}+1 \% \mathrm{H}_{2} \mathrm{O}_{2}$ & 9.03 no & 10.76 mno & 14.58 jkl & $16.85 \mathrm{~g}-\mathrm{j}$ & 12.80 & FG \\
\hline $4 \% \mathrm{CaCl}_{2}+2 \% \mathrm{H}_{2} \mathrm{O}_{2}$ & $\begin{array}{|ll|}8.60 & \text { o } \\
\end{array}$ & 10.12 no & $13.44 \mathrm{klm}$ & 15.70 ijk & 11.96 & $\mathbf{G}$ \\
\hline Means & 10.03 D & $14.78 \mathrm{C}$ & 18.85 B & $22.27 \mathrm{~A}$ & & \\
\hline
\end{tabular}

Means followed by the same letters within pre-storage applications, storage periods and their interactions in each season are not significantly different at level $P \leq 0.05$ according to DMRT.

seasons, respectively. On the contrary, this treatment had the highest TA $(0.93 \& 0.86 \mathrm{~g}$ citric acid/100 $\mathrm{ml}$ juice) in the first and second seasons, respectively. On the other hand, untreated guava fruits (control) recorded the highest fruit contents of TSS $(11.08 \& 11.27$ ${ }^{\circ}$ Brix) and TSS/TA ratio (19.26 \& 22.29) in 2015 and 2016 seasons, respectively, and also recorded the lowest fruit content of TA $(0.63 \&$ $0.56 \mathrm{~g}$ citric acid/100 ml juice) in the first and second seasons, respectively.

The interaction effects between pre-storage treatments and storage periods showed significant difference at $p \leq 0.05$ for 'Etmany' guava fruits contents of TSS, TA and TSS/TA ratio during the two seasons in this study.

Citric acid is the major organic acid in guava fruits, which is an important parameter in maintaining the quality of fruits and a rapid reduction in acidity hasted fruit senescence (Hiwale \& Singh, 2003). The increase of TA as well as the decrease of TSS and TSS/TA ratio contents of 'Etmany' guavas dipping in $\mathrm{CaCl}_{2}$, $\mathrm{H}_{2} \mathrm{O}_{2}$ and their combinations especially those treated with $4 \% \mathrm{CaCl}_{2}$ plus 1 or $2 \% \mathrm{H}_{2} \mathrm{O}_{2}$ might be due to lowering respiration rate, ethylene production and hydrolytic enzymes as mentioned by Khandaker et al. (2012) and Reena (2016).
Thereby, these applications delayed ripening and physiological senescence of the fruits during storage as compared to the untreated fruits (control).

In addition, the progressive decline of guava fruit content in TA with the advancement of storage period storage might be due to utilization of organic acids in respiration process and conversion of acids into salts and sugars by the enzymes (Hiwale \& Singh, 2003). On the other hand, the increase of guavas content in TSS with prolonging of storage period could be attributed to the hydrolysis of starch into sugars and an increase in water soluble galacturonic acids from the degradation of pectic substances by hydrolytic enzymes (Jain et al., 2003). Whereas, slight decline of fruits content in TSS at the end of storage period due to utilization of soluble solids in respiratory processes (Bashir \& AbuGoukh, 2003).

These results are in agreement with the earlier reports of Hiwale \& Singh (2003), Shaaban (2006), Kumar et al. (2012) and Reena (2016). They noted that soaking guava fruits in $\mathrm{CaCl}_{2}$ before storage resulted in delaying the increase of fruit content in TSS and showed the higher flesh acid content as compared to the untreated fruits (control) during storage and 
shelf life. Similarly, higher guava fruits content of TA was achieved by $\mathrm{CaCl}_{2}$ application as compared to the control (Mahajan et al., 2011; Phani Phani et al., 2016 and Dohre, 2016). In addition, these authors mentioned that TSS increased during storage followed by a decline at the end of storage period, while TA decreased gradually and significantly with the advancement of storage period. In addition, our results fall in line with the findings of Dohre (2016) who mentioned that, treated guava fruits with $\mathrm{CaCl}_{2}$ significantly delayed the increase of TSS/TA ratio during storage in comparison to untreated fruits, and this ratio increased with the advancement of storage period. Moreover, the same results were reported by Ismail et al. (2010) who reported that dipping 'Maamoura' guavas in $2 \% \mathrm{CaCl}_{2}$ or $2 \% \mathrm{H}_{2} \mathrm{O}_{2}$ four minutes individually or plus fumigation with lemon grass oil delayed the increase of fruit content in TSS as compared to the untreated fruits during cold storage at $8^{\circ} \mathrm{C}$ for 15 days. In addition, these results are in accordance with those reported by Mohamed et al. (2016) who claimed that, dipping 'Valencia' oranges in $2 \% \mathrm{H}_{2} \mathrm{O}_{2}$ for five minutes significantly slowed the increase of TSS and TSS/TA ratio maintained TA than the control during cold storage at $8^{\circ} \mathrm{C}$ for fifteen weeks. Furthermore, fruit contents of TSS and TSS/TA ratio increased with the progress of storage period, while TA decreased.

\section{Conclusion}

Generally, the experiment conducted here indicated that, the pre-storage application of 2 or $4 \% \mathrm{CaCl}_{2}$ and 1 or $2 \% \mathrm{H}_{2} \mathrm{O}_{2}$ individually or in combinations of 'Etmany' guavas significantly reduced the deterioration of physical and chemical characteristics of fruits as compared to the control treatment during cold storage and shelf life. Dipping 'Etmany' guava fruits in 4\% $\mathrm{CaCl}_{2}$ plus 1 or $2 \% \mathrm{H}_{2} \mathrm{O}_{2}$ for five minutes before storage are the promising methods for delaying ripening and senescence processes of fruits. Furthermore, these applications will suppress postharvest deterioration of external and internal fruit quality as well as extend the storage life of this cultivar up to 21 days at $8^{\circ} \mathrm{C}$ plus two days shelf life at ambient temperature $\left(18-23^{\circ} \mathrm{C}\right)$.

\section{REFERENCES}

Anonymous (2016). Ministry of Agriculture and Land Reclamation, Economic Affairs Sector, Bulletin of The Agricultural Statistics.

A.O.A.C. (2000). Official Methods of Analysis.
(17 $7^{\text {th }}$ Edition) Association of Official Analytical Chemists, Washington, D.C, USA. 16-20.

Bashir H.A. and Abu-Goukh A.A. (2003). Compositional changes during guava fruit ripening. Food Chem., 80 (4), 557-563.

Boora R.S., Dhaliwal H.S. and Arora N.K. (2016). Crop regulation in guava-A review. Agric. Rev., 37 (1): 1-9.

Botelho R.V., Souza N.L. and Peres N.A.R. (2000). Effect of the postharvest treatment with calcium chloride by the temperature differential method on the control of Colletotrichum gloeosporioides in guavas 'Branca de Kumagii'. Summa Phytopathol., 26 (2), 268-271.

Conway W.S., Sams C.E. and Kelman A. (1994). Enhancing the natural resistance of plant tissue to postharvest diseases through calcium applications. Hort Sci., 29: 751754.

Dat J., Vandenabeele S., Vranova E., Montagu M., Inze D. and Breusegem F. V. (2000). Dual action of the active oxygen species during plant stress responses. Cellular and Mol. Life Sci., 57: 779-795.

Davey M.W., Van Montagu M., Inze D., Sanmartin M., Kanellis A. and Smirnoff N. (2000). Plant L-ascorbic acid: chemistry, function, metabolism, bioavailability and effects of processing. $J$. Sci. Food and Agric., 80: 825-860.

Demarty M., Morvan C. and Thellier M. (1984) .Calcium and the cell wall. Plant Cell Environ., 7, 441-448.

Dhillon B.S. and Kaur S. (2013). Effect of postharvest application of calcium chloride on storage life of mango var. Dushehari fruits. Hort Flora Res. Spect., 2 (3), 265-267.

Diana M. A.B., Rico D., Frias J.M., Barat J.M., Henehan G.T.M. and Barry-Ryan C. (2007). Calcium for extending the shelf life of fresh whole and minimally processed fruits and vegetables: a review. Trends in Food Sci. Tech., 18: 210-218.

Dohre R.D. (2016). Influence of postharvest treatments on shelf life and quality of Guava (Psidium guajava L) cv. Allahabad Safeda. M.Sc. Thesis. Department of Fruit Science, Rajmata Vijayaraje Scindia Krishi Vishwa Vidyalaya, Gwalior, KNK College of Horticulture, Mandsaur (M.P.)458001, India.

Hiwale S. S. and Singh S. P. (2003). Prolonging 
shelf life of guava (Psidium guajava L.). Indian J. Hort., 60: 1-9.

Ismail S. Z., Khandaker M. M., Mat N. and Boyce A. N. (2015). Effects of hydrogen peroxide on growth, development and quality of fruits: a review. J. Agron., 14 (4): 331-336.

Ismail O. M., Abd El-Moniem E. A. A., AbdAllah A.S.E. and El-Naggar M. A. A. (2010). Influence of some postharvest treatments on guava fruits. Agric. and Biol. J. North Amer., 1 (6), 1309-1318.

Jain N., Dhawan K., Malhotra S. and Singh R. (2003). Biochemistry of fruit ripening in guava (Psidium guajava L.) compositional and enzymatic changes. Plant Foods for Human Nutr., 58: 309-315.

Juven B. J. and Pierson M. D. (1996). Antibacterial effects of hydrogen peroxide and methods for its detection and quantitation. J. Food Protect., 59 (11): 1233-1241.

Khandaker M. M., Boyce A. N. and Osman N. (2012). The influence of hydrogen peroxide on the growth, development and quality of wax apple (Syzygium samarangense, var. Jambu Madu) fruits. Plant Physiol. Biochem., 53: 101-110.

Kumar R., Lal S. and Mishra K. K. (2012). Effect of postharvest calcium treatments on shelf life of guava cv. Sardar. HortFlora Res. Spect., 1 (4): 344-347.

Mahajan B.V.C., Ghuman B.S. and Bons H.K. (2011). Effect of postharvest treatment of calcium chloride and gibbrellic acid on storage behaviour and quality of guava fruits. J. Hort. Sci. Ornament. Plants, 3: $38-42$.

McGuire R.G. (1992). Reporting of objective color measurements. Hort Sci., 27 (12): 1254-1255.

Mohamed M. A. A., Abd El-khalek A. F., Elmehrat H. G. and Mahmoud G. A. (2016). Nitric oxide, oxalic acid and hydrogen peroxide treatments to reduce decay and maintain postharvest quality of Valencia orange fruits during cold storage. Egypt. J. Hort., 43 (1): 137-161.
M-STAT (1993). A microcomputer program for the design, arrangement and analysis of agronomic research experiments. Michigan State University,USA.

Pérez-Gutiérrez R.M., Mitchell S. and VargasSolis R. (2008). Psidium guajava: a review of its traditional uses, phytochemistry and pharmacology. J. Ethnopharmacol., 117 (1), 1-27.

Phani D., V., Sekhar C.R., Srihari D. and Sankar S. A. (2016). Guava fruit quality and storability as influenced by harvest maturity and postharvest application of calcium salts. Plant Arch., 16 (1): 174182.

Reena D.M.S.R. (2016). Role of gibberellic acid and calcium chloride in ripening related biochemical changes in guava (Psidium guajava L.) fruit. Ph.D Thesis. Department of Chemistry and Biochemistry, College of Basic Science and Humanities CCS, Haryana Agricultural University, Hisar125004 , India.

Reyes M.U. and Paul R.E. (1995). Effects of storage temperature and ethylene treatments of guava (Psuidium guajava $\mathrm{L}$.) fruit ripening. Postharvest Biol. Tech., 6: 357-365.

Sapers G. M. and Simmons G. F. (1998). Hydrogen peroxide disinfections of minimally processed fruits and vegetables. Food Tech., 52 (2): 48-52.

Shaaban F.K. M. (2006). Effect of some pre and postharvest treatments on storability of guava fruits. M.Sc. Thesis. Faculty of Agriculture, Ain Shams University, Cairo Egypt.

Shirzadeh E., Rabiei V. and Sharafi Y. (2011). Effect of calcium chloride $\left(\mathrm{CaCl}_{2}\right)$ on postharvest quality of apple fruits. Afr. J. Agric. Res., 6: 5139-5143.

Silva M. E., Bautista B. P. and Velasco G. M. A. (1998). Fruit development, harvest index and ripening changes of guavas produced in central Mexico. Postharvest Biol. and Tech., 13: 143-150.

Watkins C. and Harman J. (1981). Use of penetrometer to measure flesh firmness of fruit. Orchadist, N.Z., 45: 14-16. 


$$
\begin{aligned}
& \text { تأخير تدهور ثمار الجوافة الثتوية أثناء التخزين باستخدام معاملات ما بعد الحصاد } \\
& \text { بكلوريد الكالسيوم وفوق أكسيد الهيدروجين } \\
& \text { محمود على أحمد محمد ـ *أحمد فتحي عبد الخالق } \\
& \text { معهد بحوث البساتين - مركز البحوث الزر اعية ـ مصر. }
\end{aligned}
$$

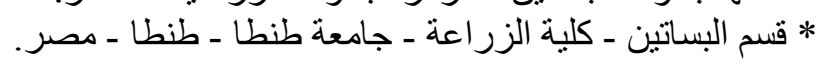

\section{ملخص}

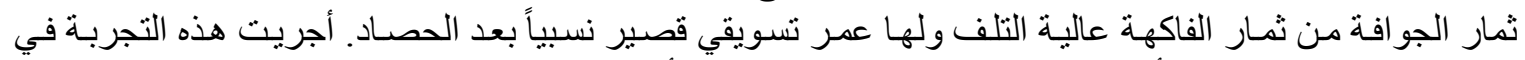

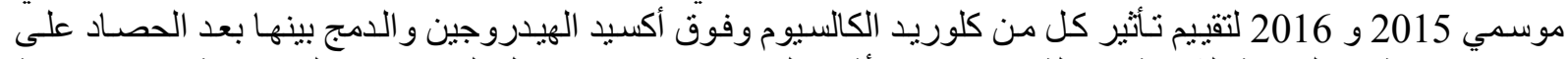

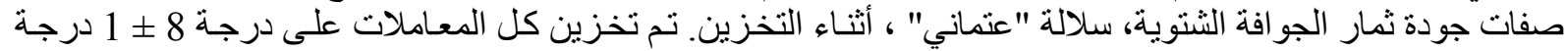

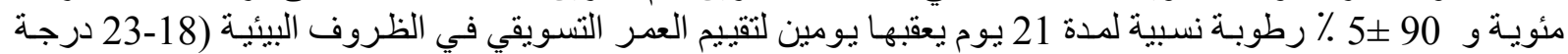

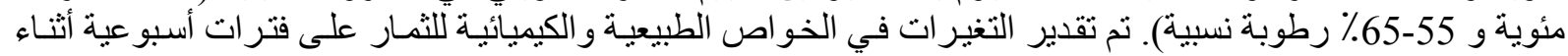

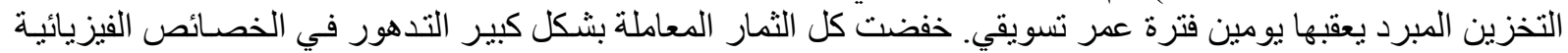

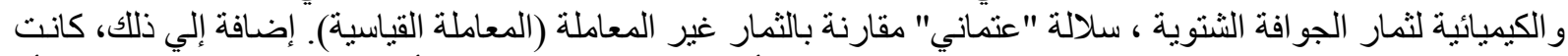

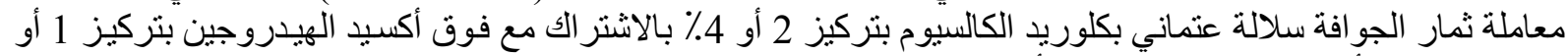

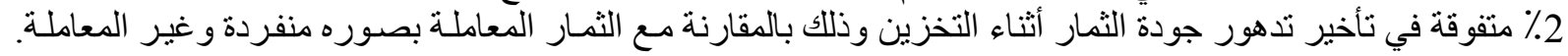

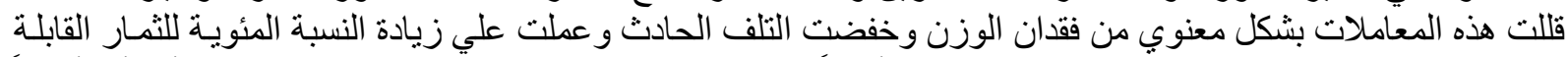

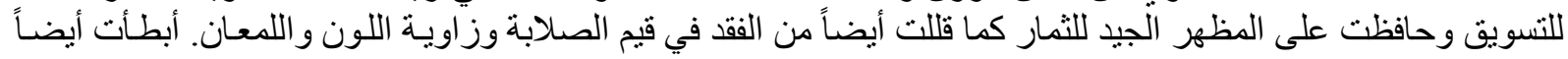

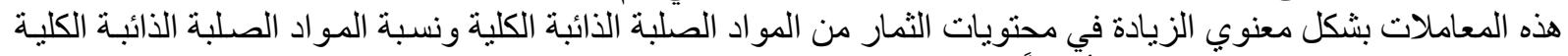

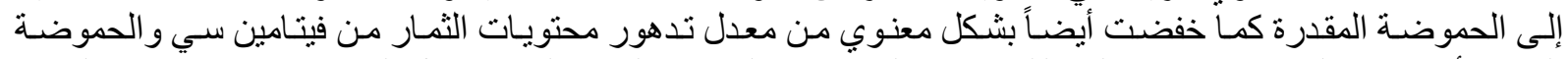

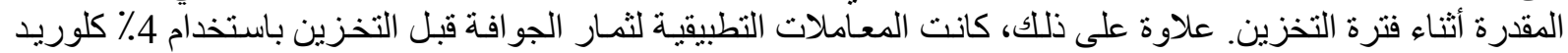

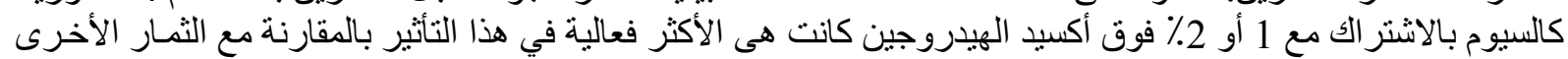

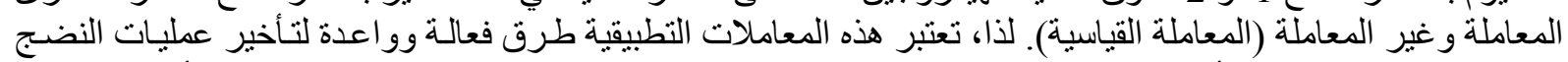

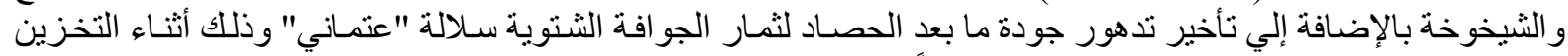

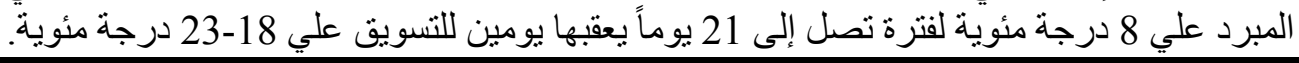

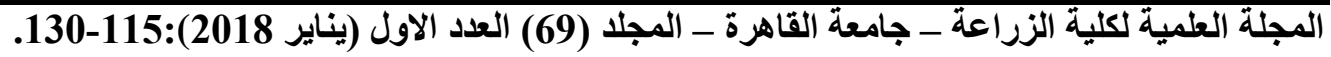

